\title{
A photon dominated region code comparison study ${ }^{\star}$
}

\author{
M. Röllig ${ }^{1,4}$, N. P. Abel ${ }^{2}$, T. Bell ${ }^{3,17}$, F. Bensch ${ }^{1}$, J. Black ${ }^{15}$, G. J. Ferland ${ }^{2}$, B. Jonkheid ${ }^{5}$, I. Kamp ${ }^{6}$, M. J. Kaufman ${ }^{7}$, \\ J. Le Bourlot ${ }^{8}$, F. Le Petit ${ }^{8,15}$, R. Meijerink ${ }^{5}$, O. Morata ${ }^{16}$, V. Ossenkopf ${ }^{4,10}$, E. Roueff ${ }^{8}$, G. Shaw ${ }^{2}$, M. Spaans ${ }^{9}$, \\ A. Sternberg ${ }^{11}$, J. Stutzki ${ }^{4}$, W.-F. Thi ${ }^{12}$, E. F. van Dishoeck ${ }^{5}$, P. A. M. van Hoof ${ }^{13}$, S. Viti ${ }^{3}$, and M. G. Wolfire ${ }^{14}$
}

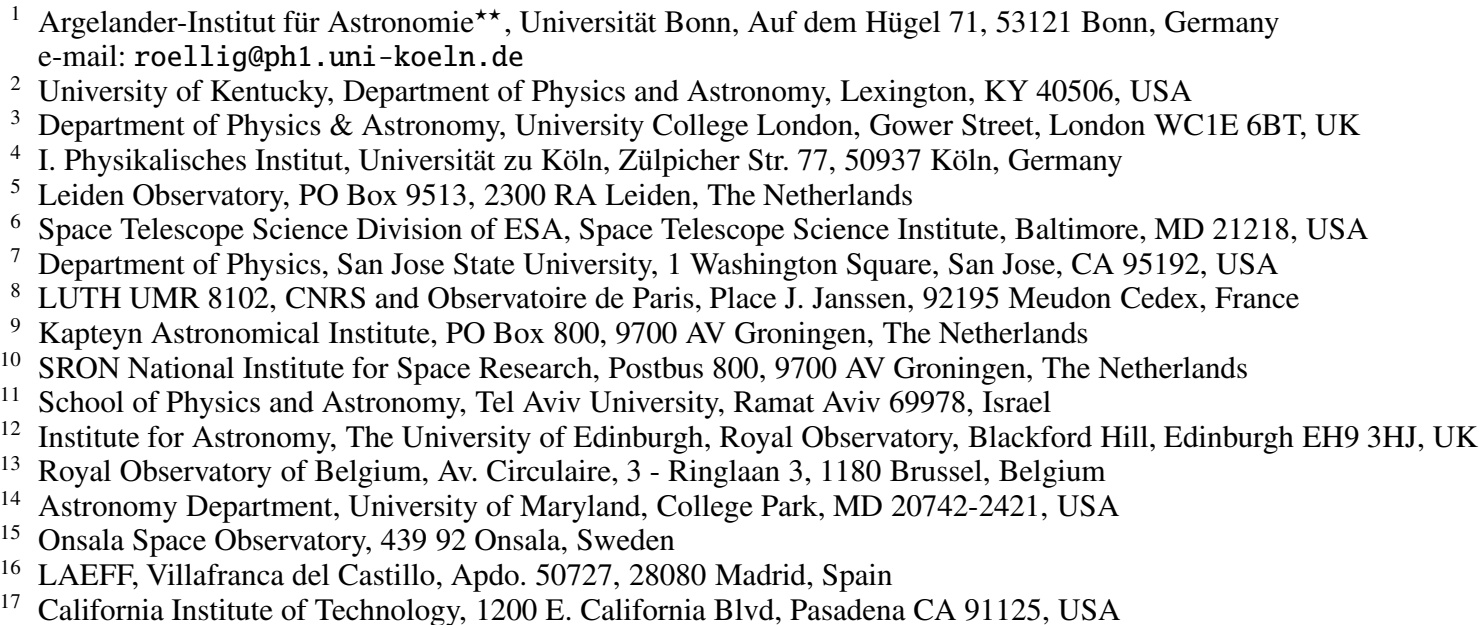

Received 27 June 2006 / Accepted 2 February 2007

\section{ABSTRACT}

\begin{abstract}
Aims. We present a comparison between independent computer codes, modeling the physics and chemistry of interstellar photon dominated regions (PDRs). Our goal was to understand the mutual differences in the PDR codes and their effects on the physical and chemical structure of the model clouds, and to converge the output of different codes to a common solution.

Methods. A number of benchmark models have been created, covering low and high gas densities $n=10^{3}, 10^{5.5} \mathrm{~cm}^{-3}$ and far ultraviolet intensities $\chi=10,10^{5}$ in units of the Draine field (FUV: $6<h v<13.6 \mathrm{eV}$ ). The benchmark models were computed in two ways: one set assuming constant temperatures, thus testing the consistency of the chemical network and photo-processes, and a second set determining the temperature self consistently by solving the thermal balance, thus testing the modeling of the heating and cooling mechanisms accounting for the detailed energy balance throughout the clouds.

Results. We investigated the impact of PDR geometry and agreed on the comparison of results from spherical and plane-parallel PDR models. We identified a number of key processes governing the chemical network which have been treated differently in the various codes such as the effect of PAHs on the electron density or the temperature dependence of the dissociation of CO by cosmic ray induced secondary photons, and defined a proper common treatment. We established a comprehensive set of reference models for ongoing and future PDR model bench-marking and were able to increase the agreement in model predictions for all benchmark models significantly. Nevertheless, the remaining spread in the computed observables such as the atomic fine-structure line intensities serves as a warning that there is still a considerable uncertainty when interpreting astronomical data with our models.
\end{abstract}

Key words. ISM: abundances - astrochemistry - ISM: clouds - ISM: general - radiative transfer - methods: numerical

\section{Introduction}

Interstellar photon dominated regions or photodissociation regions (PDRs) play an important role in modern astrophysics as they are responsible for many emission characteristics of the ISM, and dominate the infrared and sub-millimetre spectra of star formation regions and galaxies as a whole. Theoretical models addressing the structure of PDRs have been available for approximately 30 years and have evolved into advanced

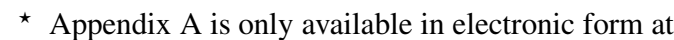
http: //www . aanda.org

$\star \star$ Founded by merging of the Sternwarte, Radiastronomisches Institut and Institut für Astrophysik und Extraterestrische Forschung. computer codes accounting for a growing number of physical effects with increasing accuracy. These codes have been developed with different goals in mind: some are geared to efficiently model a particular type of region, e.g. HII regions, protoplanetary disks, planetary nebulae, diffuse clouds, etc.; others emphasize a strict handling of the micro-physical processes in full detail (e.g. wavelength dependent absorption), but at the cost of increased computing time. Yet others aim at efficient and rapid calculation of large model grids for comparison with observational data, which comes at the cost of pragmatic approximations using effective rates rather than detailed treatment. As a result, the different models have focused on the detailed simulation of particular processes determining the structure in the main 
regions of interest while using only rough approximations for other processes. The model setups vary strongly among different model codes. This includes the assumed model geometry, their physical and chemical structure, the choice of free parameters, and other details. Consequently it is not always straightforward to directly compare the results from different PDR codes. Taking into account that there are multiple ways of implementing physical effects in numerical codes, it is obvious that the model output of different PDR codes can differ from each other. As a result, significant variations in the physical and chemical PDR structure predicted by the various PDR codes can occur. This divergence would prevent a unique interpretation of observed data in terms of the parameters of the observed clouds. Several new facilities such as Herschel, SOFIA, APEX, ALMA, and others will become available over the next years and will deliver many high quality observations of line and dust continuum emission in the sub-millimeter and FIR wavelength regime. Many important PDR tracers emit in this range ([CII] $(158 \mu \mathrm{m}),[\mathrm{OI}]$ (63 and $146 \mu \mathrm{m}),[\mathrm{CI}](370$ and $610 \mu \mathrm{m}), \mathrm{CO}(650,520, \ldots, 57.8 \mu \mathrm{m})$, $\mathrm{H}_{2} \mathrm{O}$, etc.). In order to reliably analyze these data we need a set of high quality tools, including PDR models that are well understood and properly debugged. As an important preparatory step toward these missions an international cooperation between many PDR model groups was initiated. The goals of this PDRbenchmarking were:

- to understand the differences in the different code results;

- to obtain (as much as possible) the same model output with every PDR code when using the same input;

- to agree on the correct handling of important processes;

- to identify the specific limits of applicability of the available codes.

To this end, a PDR-benchmarking workshop was held at the Lorentz Center in Leiden, Netherlands in 2004 to jointly work on these topics ${ }^{1}$. In this paper we present the results from this workshop and the results originating from the follow-up activities. A related workshop to test line radiative transfer codes was held in 1999 (see van Zadelhoff et al. 2002).

It is not the purpose of the benchmarking to present a preferred solution or a preferred code. PDRs are found in a large variety of objects and under very different conditions. To this end, it was neither possible nor desirable to develop a generic PDR code, able to model every possible PDR. Furthermore, the benchmarking is not meant to model any "real" astronomical object. The main purpose of this study is technical not physical. This is also reflected in the choice of the adopted incomplete chemical reaction network (see Sect. 4).

In Sect. 2 we briefly introduce the physics involved in PDRs, in Sect. 3 we introduce some key features in PDR modeling. Section 4 describes the setup of the benchmark calculations and Sect. 5 presents the results for a selection of benchmark calculations and gives a short review over the participating codes. In Sect. 6 we discuss the results and summarize the lessons learned from the benchmark effort. A tabular overview of the individual code characteristics is given in the Appendix.

\section{The physics of PDRs}

PDRs are traditionally defined as regions where $\mathrm{H}_{2}$-non-ionizing far-ultraviolet photons from stellar sources control the gas heating and chemistry. Any ionizing radiation is assumed to be absorbed in the narrow ionization fronts located between adjacent

\footnotetext{
${ }^{1}$ URL: http://www. lorentzcenter.nl/
}

HII regions and the PDRs ${ }^{2}$. In PDRs the gas is heated by the far-ultraviolet radiation (FUV, $6<h v<13.6 \mathrm{eV}$, from the ambient UV field and from hot stars) and cooled via the emission of spectral line radiation of atomic and molecular species and continuum emission by dust (Hollenbach \& Tielens 1999; Sternberg 2004). The FUV photons heat the gas by means of photoelectric emission from grain surfaces and polycyclic aromatic hydrocarbons (PAHs) and by collisional de-excitation of vibrationally excited $\mathrm{H}_{2}$ molecules. Additional contributions to the total gas heating comes from $\mathrm{H}_{2}$ formation, dissociation of $\mathrm{H}_{2}$, dust-gas collisions in case of dust temperatures exceeding the gas temperature, cosmic ray heating, turbulence, and from chemical heating. At low visual extinction $A_{\mathrm{V}}$ into the cloud/PDR the gas is cooled by emission of atomic fine-structure lines, mainly [OI] $63 \mu \mathrm{m}$ and [CII] $158 \mu \mathrm{m}$. At larger depths, millimeter, sub-millimeter and far-infrared molecular rotational-line cooling $\left(\mathrm{CO}, \mathrm{OH}, \mathrm{H}_{2}, \mathrm{H}_{2} \mathrm{O}\right)$ becomes important, and a correct treatment of the radiative transfer in the line cooling is critical. The balance between heating and cooling determines the local gas temperature. The local FUV intensity also influences the chemical structure, i.e. the abundance of the individual chemical constituents of the gas. The surface of PDRs is mainly dominated by reactions induced by UV photons, especially the ionization and dissociation of atoms and molecules. With diminishing FUV intensity at higher optical depths more complex species may be formed without being radiatively destroyed immediately. Thus the overall structure of a PDR is the result of a complex interplay between radiative transfer, energy balance, and chemical reactions.

\section{Modeling of PDRs}

The history of PDR modeling dates back to the early 1970's (Hollenbach et al. 1971; Jura 1974; Glassgold \& Langer 1975; Black \& Dalgarno 1977) with steady state models for the transitions from $\mathrm{H}$ to $\mathrm{H}_{2}$ and from $\mathrm{C}^{+}$to $\mathrm{CO}$. In the following years a number of models, addressing the chemical and thermal structure of clouds subject to an incident flux of FUV photons have been developed (de Jong et al. 1980; Tielens \& Hollenbach 1985; van Dishoeck \& Black 1988; Sternberg \& Dalgarno 1989; Hollenbach et al. 1991; Le Bourlot et al. 1993; Störzer et al. 1996). Additionally, a number of models, focusing on certain aspects of PDR physics and chemistry were developed, e.g. models accounting for time-dependent chemical networks, models of clumped media, and turbulent PDR models (Hill \& Hollenbach 1978; Wagenblast \& Hartquist 1988; de Boisanger et al. 1992; Bertoldi \& Draine 1996; Lee et al. 1996; Hegmann \& Kegel 1996; Spaans 1996; Nejad \& Wagenblast 1999; Röllig et al. 2002; Bell et al. 2005). Standard PDR models generally do not account for dynamical properties of gas but there are some studies that consider the advection problem rather than the steady state approach (e.g. Störzer \& Hollenbach 1998). For a more detailed review see Hollenbach \& Tielens (1999).

In order to numerically model a PDR it is necessary to compute all local properties of a cloud such as the relative abundances of the gas constituents together with their level populations, temperature of gas and dust, gas pressure, composition of dust/PAHs, and many more. This local treatment is complicated by the radiation field which couples remote parts of the cloud. The local mean radiation field, which is responsible for photochemical reactions, gas/dust heating, and excitation of molecules

2 This distinction is clearer when referring to PDRs as PhotoDissociation Regions, since molecules are hardly found in HII regions. 
depends on the position in the cloud and the (wavelength dependent) absorption along the lines of sight toward this position. This non-local coupling makes numerical PDR calculations a CPU time consuming task.

PDR modelers and observers approach the PDRs from opposite sides: PDR models start by calculating the local properties of the clouds such as the local CO density and the corresponding gas temperature and use these local properties to infer the expected global properties of the cloud like total emergent emissivities or fluxes and column densities. The observer on the other hand starts by observing global features of a source and tries to infer the local properties from that. The connection between local and global properties is complex and not necessarily unambiguous. Large variations e.g. in the $\mathrm{CO}$ density at the surface of the cloud may hardly affect the overall CO column density due to the dominance of the central part of the cloud with a high density. If one is interested in the total column density it does not matter whether different codes produce a different surface $\mathrm{CO}$ density. For the interpretation of high- $J \mathrm{CO}$ emission lines, however, different $\mathrm{CO}$ densities in the outer cloud layers make a huge difference since high temperatures are required to produce high- $J$ CO fluxes. Thus, if different PDR model codes deviate in their predicted cloud structures, this may affect the interpretation of observations and may prevent inference of the "true" structure behind the observed data. To this end it is very important to understand the origin of present differences in PDR model calculations. Otherwise it is impossible to rule out alternative interpretations. The ideal situation, from the modelers point of view, would be a complete knowledge of the true local structure of a real cloud and their global observable properties. This would easily allow us to calibrate PDR models. Since this case is unobtainable, we take one step back and apply a different approach: If all PDR model codes use exactly the same input and the same model assumptions they should produce the same predictions.

Because of the close interaction between chemical and thermal balance and radiative transfer, PDR codes typically iterate through the following computation steps: 1) solve the local chemical balance to determine local densities; 2) solve the local energy balance to estimate the local physical properties like temperatures, pressures, and level populations; 3 ) solve the radiative transfer; 4) for finite models it is necessary to successively iterate steps 1)-3). Each step requires a variety of assumptions and simplifications. Each of these aspects can be investigated to great detail and complexity (see for example van Zadelhoff et al. (2002) for a discussion of NLTE radiative transfer methods), but the explicit aim of the PDR comparison workshop was to understand the interaction of all computation steps mentioned above. Even so it was necessary to considerably reduce the model complexity in order to disentangle cause and effect.

\subsection{Description of sensitivities and pitfalls}

Several aspects of PDR modeling have shown the need for detailed discussion, easily resulting in misleading conclusions if not treated properly.

\subsubsection{Model geometry}

Two common geometrical setups of model PDRs are shown in Fig. 1. Most PDR models feature a plane-parallel geometry, illuminated either from one side or from both sides. This geometry naturally suggests a directed illumination, perpendicular to the cloud surface. This simplifies the radiative transfer problem
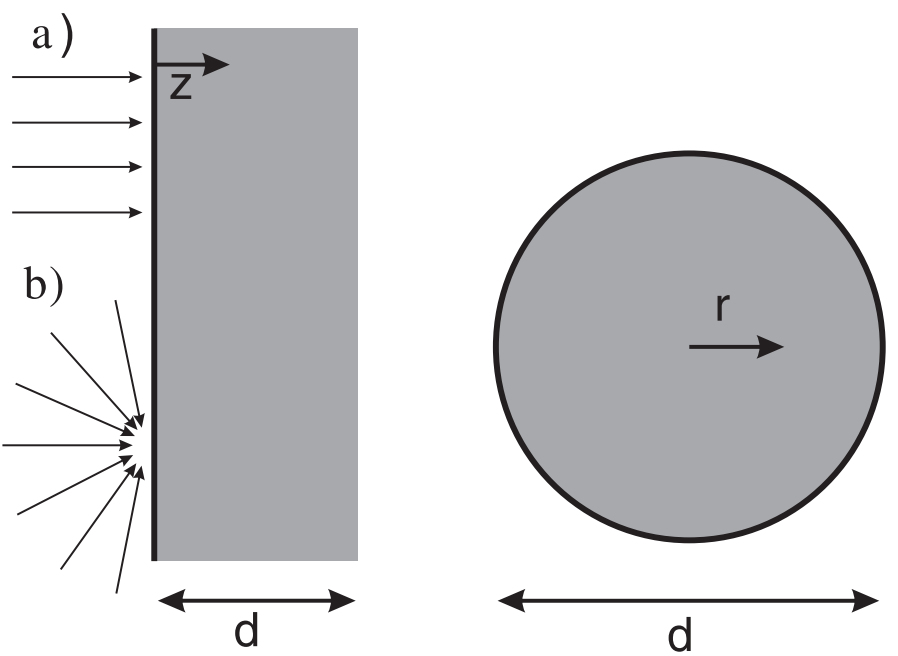

Fig. 1. Common geometrical setups of a model PDR. The surface of any plane-parallel or spherical cloud is illuminated either a) uni-directional or b) isotropically.

significantly, since it is sufficient to account for just one line of sight, if we ignore scattering out of the line of sight (Flannery et al. 1980). Since most plane-parallel PDR models are infinite perpendicular to the cloud depth $z$ it is also straightforward to account for an isotropic FUV irradiation within the pure 1-D formalism. For a spherical geometry one can exploit the model symmetry only for a FUV field isotropically impinging onto the cloud. In finite plane-parallel and spherical models iterations over the depth/radial structure are mandatory because radiation is coming from multiple directions, passing through cloud elements for which the physical and chemical structure and hence opacities have not been calculated in the same iteration step. To account for this "backside" illumination it is essential to iterate on the radiation field.

The most important quantity describing the radiation field in PDR models is the local mean intensity (or alternatively the energy density) as given by:

$J_{v}=\frac{1}{4 \pi} \int I_{v} \mathrm{~d} \Omega\left[\mathrm{erg} \mathrm{cm}^{-2} \mathrm{~s}^{-1} \mathrm{~Hz}^{-1} \mathrm{sr}^{-1}\right]$

with the specific intensity $I_{v}$ being averaged over the solid angle $\Omega$. Note that when referring to the ambient FUV in units of Draine $\chi$ (Draine 1978) or Habing $G_{0}$ (Habing 1968) fields, these are always given as averaged over $4 \pi$. If we place a model cloud of sufficient optical thickness in such an average FUV field, the resulting local mean intensity at the cloud edge is half the value of that without the cloud.

The choice between directed and isotropic FUV fields directly influences the attenuation due to dust. In the unidirectional case the FUV intensity along the line of sight is attenuated according to $\exp \left(-\tau_{v}\right)$, where $\tau_{v}$ is the optical depth of the dust at frequency $v$. For pure absorption the radiative transfer equation becomes:

$\mu \frac{\mathrm{d} I_{v}(\mu, x)}{\mathrm{d} x}=-\kappa_{v} I_{v}(\mu, x)$.

with the cosine of the radiation direction $\mu=\cos \Theta$, the cloud depth $x$, and the absorption coefficient $\kappa_{v}$, with the simple solution $J_{v} / J_{v, 0}=\exp \left(-\tau_{v} \mu\right)$ for a semi-infinite cloud. For the 


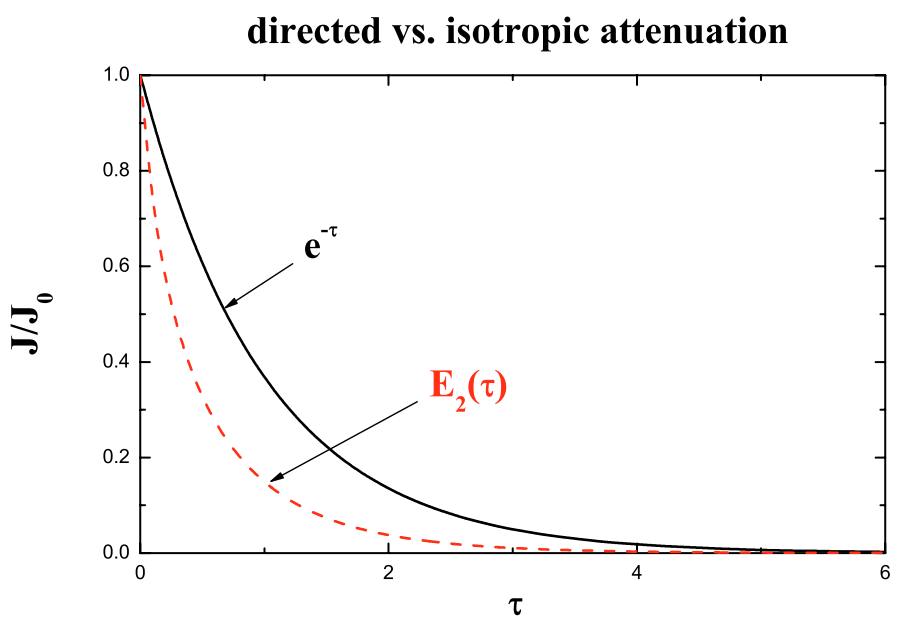

Fig. 2. Comparison of attenuation of the mean intensity for the case of an uni-directional and isotropically illuminated medium. The solid line gives the attenuation due to uni-directional illumination, while the dashed line gives the attenuation for an isotropic FUV radiation where $\tau$ means the optical depth perpendicular to the surface of the cloud.

isotropic case, $I_{v, 0}(\mu)=J_{v, 0}=$ const., integration of Eq. (2) leads to the second order exponential integral:

$J_{v} / J_{v, 0}=E_{2}\left(\tau_{v}\right)=\int_{0}^{1} \frac{\exp \left(-\tau_{v} \mu\right)}{\mu^{2}} \mathrm{~d} \mu$.

As seen in Fig. 2 the attenuation with depth in the isotropic case is significantly different from the uni-directional case. A common way to describe the depth dependence of a particular quantity in PDRs is to plot it against $A_{\mathrm{V}}$, which is a direct measure of the traversed column of attenuating material. In order to compare the uni-directional and the isotropic case it is necessary to rescale them to the same axis. It is possible to define an effective $A_{\mathrm{V}, \text { eff }}=-\ln \left[E_{2}\left(A_{\mathrm{V}} k\right)\right] / k$ with $k=\tau_{\mathrm{UV}} / A_{\mathrm{V}}$ in the isotropic case, where $A_{\mathrm{V}}$ is the attenuation perpendicular to the surface and $\mathrm{UV}$ is in the range $6<h v<13.6$. In this paper all results from spherical models are scaled to $A_{\mathrm{V} \text {,eff }}$. Figure 3 demonstrates the importance of scaling results to an appropriate $A_{\mathrm{V}}$ scale. It shows the local $\mathrm{H}_{2}$ photo-dissociation rate for two different FUV illumination geometries. The solid line represents a standard uni-directional illumination perpendicular to the cloud surface as given in many standard plane-parallel PDR codes. The dashed line is the result from an isotropic illumination plotted against the standard "perpendicular" $A_{\mathrm{V}}$. The offset to the unidirectional case is significant. After rescaling to an appropriate $A_{\mathrm{V}, \text { eff }}$ both model results are in good agreement. Please note, that in general it is not possible to achieve perfect agreement as there is a spectrum involved with a spread of $k$ values across the UV.

The attenuation of FUV radiation is additionally complicated if we account for dust scattering. For a full treatment by Legendre polynomials see Flannery et al. (1980). In case of small scattering angles $g=\langle\cos \theta\rangle \approx 1$ the scattering can be approximated by an effective forward attenuation $\tau(1-\omega)$, where $\omega$ is the scattering albedo. Thus, more material is needed to obtain the same attenuation as in the case without scattering. Hence a proper scaling of $A_{\mathrm{V}}$ is necessary. In case of clumped gas this becomes even more complex. The presence of stochastic density fluctuations leads to a substantial reduction of the effective optical depth as demonstrated by Hegmann \& Kegel (2003). All this has to be considered when calculating the photodissociation and photoionization rates, when the attenutation with depth is represented by simple exponential forms, $\exp \left(-k_{i} A_{\mathrm{V}}\right)$ (e.g.

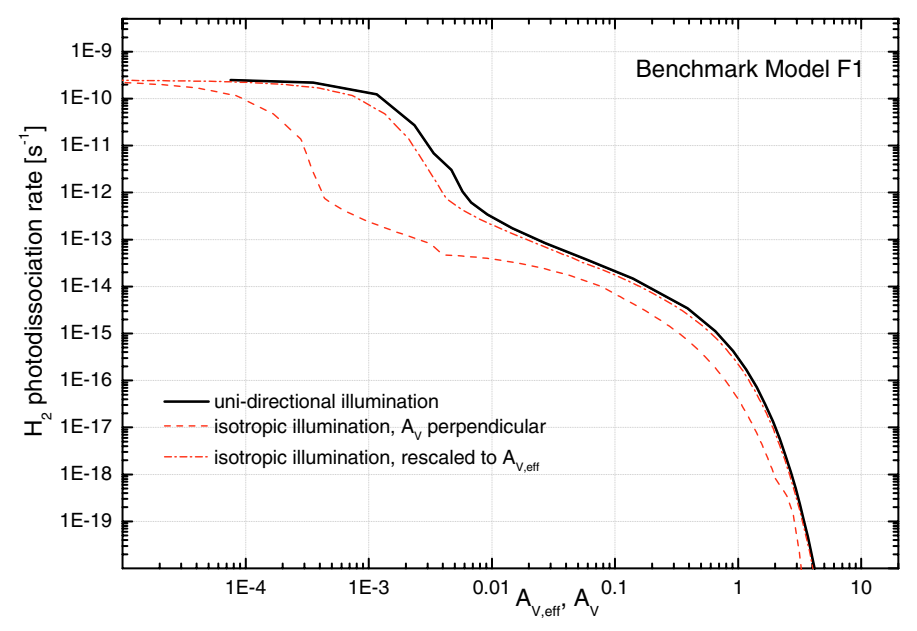

Fig. 3. $\mathrm{H}_{2}$ photo-dissociation rates resulting from uni-directional FUV illuminated clouds compared to an isotropic illumination. The results from isotropic models are plotted vs. the perpendicular $A_{\mathrm{V}}$ and vs. $A_{\mathrm{V}, \text { eff }}$.

van Dishoeck 1988; Roberge et al. 1991), where the factor $k_{i}$ accounts for the wavelength dependence of the photoprocess $i^{3}$.

\subsubsection{Chemistry}

PDR chemistry has been addressed in detail by many authors (Tielens \& Hollenbach 1985; van Dishoeck \& Black 1988; Hollenbach et al. 1991; Fuente et al. 1993; Le Bourlot et al. 1993; Jansen et al. 1995; Sternberg \& Dalgarno 1995; Lee et al. 1996; Bakes \& Tielens 1998; Walmsley et al. 1999; Savage \& Ziurys 2004; Teyssier et al. 2004; Fuente et al. 2005; Meijerink \& Spaans 2005). These authors discuss numerous aspects of PDR chemistry in great detail and give a comprehensive overview of the field. Here we repeat some crucial points in the chemistry of PDRs in order to motivate the benchmark standardization and to prepare the discussion of the benchmark result.

In PDRs photoprocesses are very important due to the high FUV intensity, as well as reactions with abundant hydrogen atoms. The formation and destruction of $\mathrm{H}_{2}$, heavily influenced by the FUV field, is of major importance for the chemistry in PDRs. $\mathrm{H}_{2}$ forms on grain surfaces, a process which crucially depends on the temperatures of the gas and the grains (Hollenbach \& Salpeter 1971; Cazaux \& Tielens 2004), which themselves depend on the local cooling and heating, governed by the FUV. The photo-dissociation of $\mathrm{H}_{2}$ is a line absorption process and, thus is subject to effective shielding (van Dishoeck \& Black 1988). This leads to a sharp transition from atomic to molecular hydrogen once the $\mathrm{H}_{2}$ absorption lines are optically thick. The photodissociation of $\mathrm{CO}$ is also a line absorption process, additionally complicated by the fact that the broad $\mathrm{H}_{2}$ absorption lines overlap with $\mathrm{CO}$ absorption lines. Similar to $\mathrm{H}_{2}$ this leads to a transition from atomic carbon to $\mathrm{CO}$. For $A_{\mathrm{V}}<1$ carbon is predominantly present in ionized form. For an assumed FUV field of $\chi=1, \mathrm{CO}$ is formed at about $A_{\mathrm{V}} \approx 2$. This results in the typical PDR stratification of $\mathrm{H} / \mathrm{H}_{2}$ and $\mathrm{C}^{+} / \mathrm{C} / \mathrm{CO}$. The depth of this transition zone depends on the physical parameters but also on the contents of the chemical network: for example the inclusion of PAHs into the chemical balance calculations shifts the $\mathrm{C}^{+}$to C transition to smaller $A_{\mathrm{V} \text {,eff }}$ (e.g. Lepp \& Dalgarno 1988; Bakes \& Tielens 1998).

\footnotetext{
3 In this context the term photoprocess refers to either photodissociation or photoionization.
} 


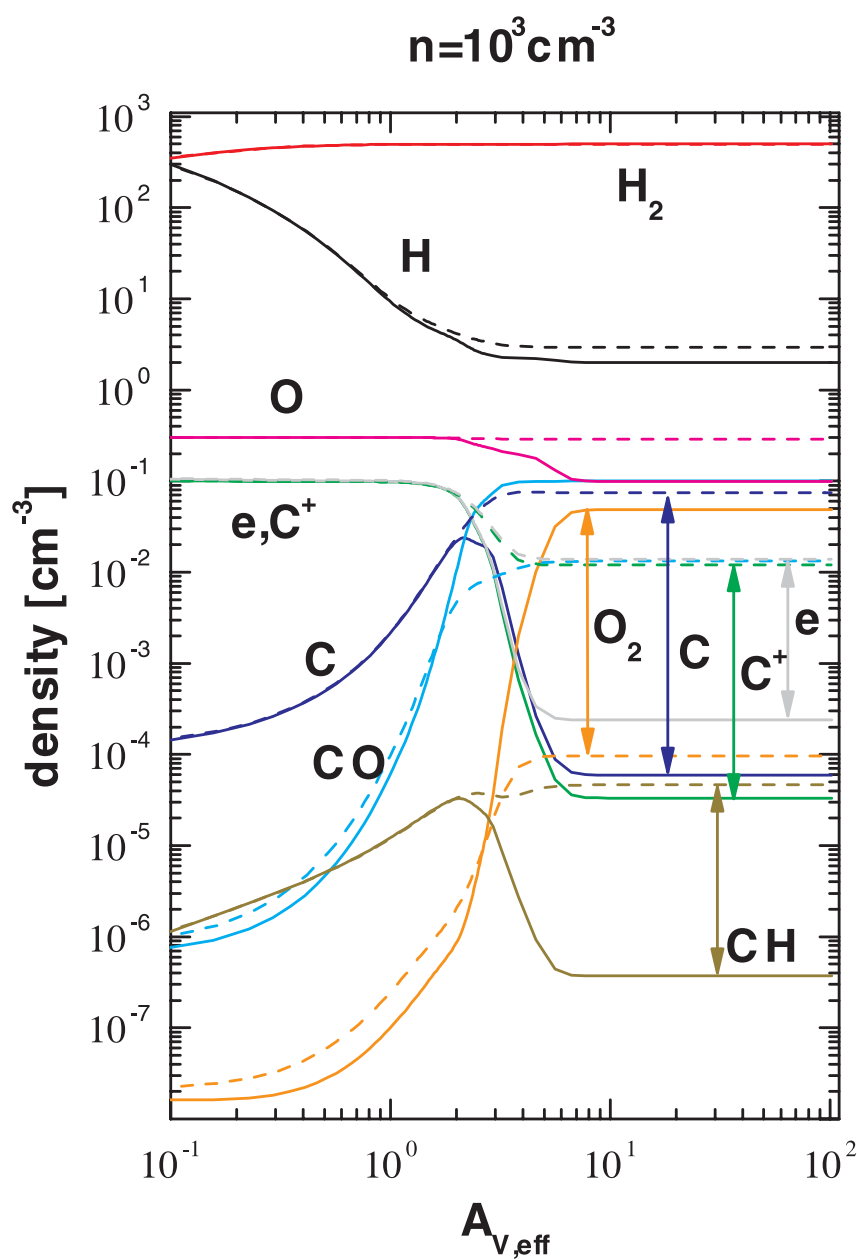

$\mathrm{n}=10^{4} \mathrm{~cm}^{-3}$

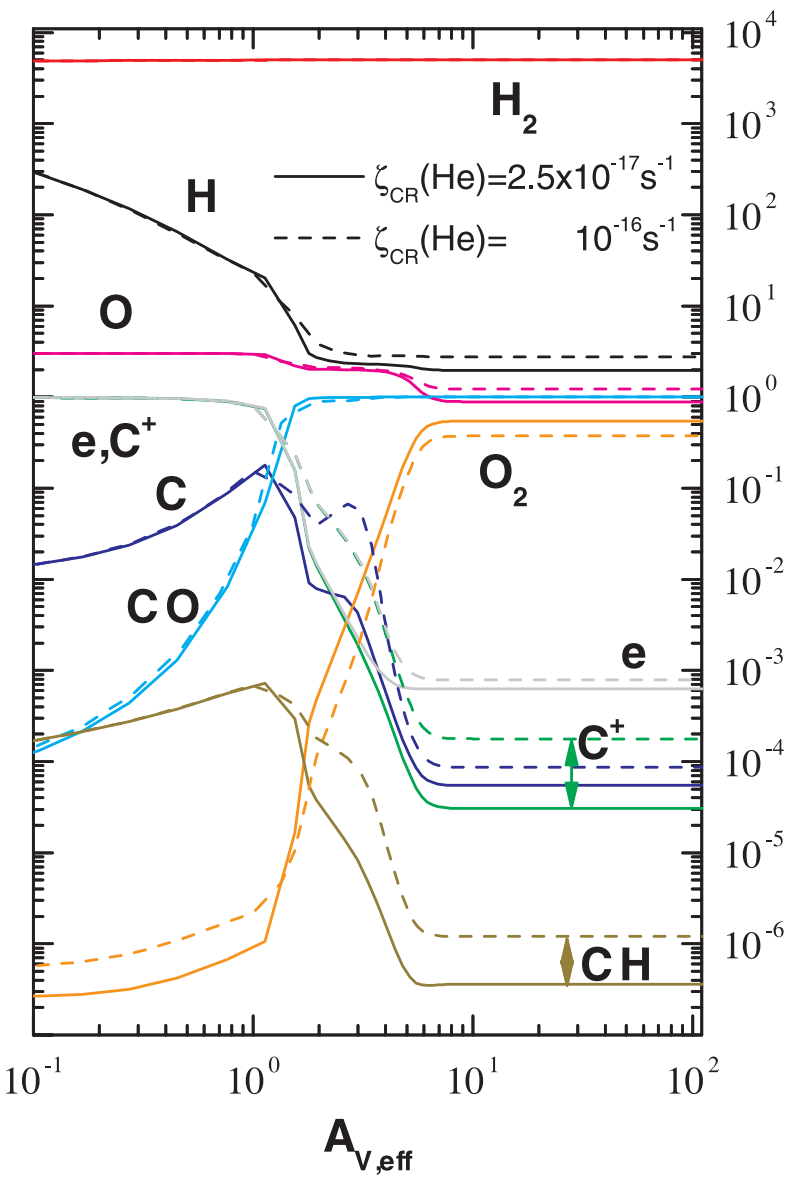

Fig. 4. The influence of the cosmic ray ionization rate on the chemical structure of a model cloud. The left panel shows results for Model F1 $\left(n=10^{3} \mathrm{~cm}^{-3}, \chi=10\right)$, the right panel gives results for 10 times higher densities $\left(n=10^{4} \mathrm{~cm}^{-3}, \chi=10\right)$. The solid lines give the results for a cosmic ray ionization rate of Helium, enhanced by a factor 4, the dashed lines are for the lower ionization rate. The different colors denote different chemical species. The most prominent differences are highlighted with colored arrows.

The solution of the chemical network itself covers the destruction and formation reactions of all chemical species considered. For each included species $i$ this results in a balance equation of the form:

$$
\begin{aligned}
\frac{\mathrm{d} n_{i}}{\mathrm{~d} t}= & \sum_{j} \sum_{k} n_{j} n_{k} R_{j k i}+\sum_{l} n_{l} \zeta_{l i} \\
& -n_{i}\left(\sum_{l} \zeta_{i l}+\sum_{l} \sum_{j} n_{j} R_{i j l}\right) .
\end{aligned}
$$

Here $n_{i}$ denotes the density of species $i$. The first two terms cover all formation processes while the last two terms account for all destruction reactions. $R_{j k i}$ is the reaction rate coefficient for the reaction $\mathrm{X}_{j}+\mathrm{X}_{k} \rightarrow \mathrm{X}_{i}+\ldots$ (X stands for species $\left.\mathrm{X}\right)$, $\zeta_{i l}$ is the local photo-destruction rate coefficient for ionization or dissociation of species $\mathrm{X}_{i}+h v \rightarrow \mathrm{X}_{l}+\ldots$, either by FUV photons or by cosmic ray (CR) induced photons, and $\zeta_{l i}$ is the local formation rate coefficient for formation of $\mathrm{X}_{i}$ by photodestruction of species $\mathrm{X}_{l}$. For a stationary solution one assumes $\mathrm{d} n_{i} / \mathrm{d} t=0$, while non-stationary models solve the differential Eq. (4) in time. The chemical network is a highly non-linear system of equations. Hence it is not self-evident that a unique solution exists at all, multiple solution may be possible as demonstrated e.g. by Le Bourlot et al. (1993) and Boger \& Sternberg (2006).
They showed that bistability may occur in gas-phase models (neglecting dust chemistry) of interstellar dark clouds in a narrow parameter range of approximately $10^{3} \mathrm{~cm}^{-3} \gtrsim n / \zeta_{-17} \gtrsim$ $10^{2} \mathrm{~cm}^{-3}$ with the cosmic-ray ionization rate of molecular hydrogen $\zeta_{\mathrm{CR}} \equiv 10^{-17} \zeta_{-17} \mathrm{~s}^{-1}$. Within this range the model results may depend very sensitively on variations of input parameters such as $\zeta_{\mathrm{CR}}$ or the $\mathrm{H}_{3}^{+}$dissociative recombination rate. To demonstrate this we show the influence of varying ionization rates in Fig. 4. The left panel gives abundance profiles for benchmark model F1 $\left(n=10^{3} \mathrm{~cm}^{-3}, \chi=10\right)$ the right panel shows a similar model but with higher density $\left(n=10^{4} \mathrm{~cm}^{-3}\right)$. The higher density was chosen to make sure that we are outside the bistability regime. The solid lines in both panels are for a cosmic ray helium ionization rate of $\zeta_{\mathrm{CR}}(\mathrm{He})=2.5 \times 10^{-17} \mathrm{~s}^{-1}$, the dashed lines denote an ionization rate increased by a factor four. Different colors denote different chemical species. The most prominent differences are highlighted with colored arrows. The factor four in $\zeta_{\mathrm{CR}}(\mathrm{He})$ results in differences in density up to three orders of magnitude in the lower density case! A detailed analysis shows that the strong abundance transitions occur for $\zeta_{\mathrm{CR}}(\mathrm{He})>8 \times 10^{-17} \mathrm{~s}^{-1}$. This highly non-linear behavior disappears if we leave the critical parameter range as demonstrates in the right panel of Fig. 4. Boger \& Sternberg (2006) emphasize that this effect is a property of the gas phase chemical network, and is damped if gas-grain processes such as grain 
assisted recombination of the atomic ions are introduced (see also Shalabiea \& Greenberg 1995). They conclude that the bistability phenomenon probably does not occur in realistic dusty interstellar clouds while Le Bourlot (2006) argues that what matters for bistability is not the number of neutralisation channels but the degree of ionisation and that bistability may occur in interstellar clouds. They suggest this could be one of the possible reasons of the non detection of $\mathrm{O}_{2}$ by the ODIN satellite (Viti et al. 2001). Yet, another possible explanation for the absence of $\mathrm{O}_{2}$ is freeze-out onto dust. However it is clear that bistability is a real property of interstellar gas-phase networks and not just a numerical artifact. Furthermore it is important to emphasize that standard PDR models may react very sensitively on the variation of input parameters (e.g. $\zeta_{\mathrm{CR}}$, the $\mathrm{H}_{2}$ formation rate, the PAH content of the model cloud, etc.) and one has to be careful in the interpretation of surprising model signatures.

The numerical stability and the speed of convergence may vary significantly over different chemical networks. Three major questions have to be addressed:

1. which species $i$ are to be included?

2. which reactions are to be considered?

3. which reaction rate coefficients are to be applied?

A general answer to question 1 cannot be given, since this depends on the field of application. In steady state one has to solve a system of $M$ nonlinear equations, where $M$ is the number of included species, thus the complexity of the problem scales with the number of species $\left(\propto N^{2} \ldots N^{3}\right)$ rather than with the number of chemical reactions. Nowadays CPU time is not a major driver for the design of chemical networks. Nevertheless, in some cases a small network can give similar results as a big network. Several studies have shown that very large networks may include a surprisingly large number of "unimportant" reactions, i.e. reactions that may be removed from the network without changing the chemical structure significantly (MarkwickKemper 2005; Wakelam et al. 2005a). It is more important to identify crucial species not to be omitted, i.e. species that dominate the chemical structure under certain conditions. A well known example is the importance of sulfur for the formation of atomic carbon at intermediate $A_{\mathrm{V}}$ where the charge transfer reaction $\mathrm{S}+\mathrm{C}^{+} \rightarrow \mathrm{C}+\mathrm{S}^{+}$constitutes an additional production channel for atomic carbon, visible in a second rise in the abundance of C (Sternberg \& Dalgarno 1995). In these benchmarking calculations, sulfur was not included in order to minimize model complexity, in spite of its importance for the PDR structure.

Regarding question 2 a secure brute force approach would be the inclusion of all known reactions involving all chosen species, under the questionable assumption that we actually know all important reactions and their rate coefficients. This assumption is obviously invalid for grain surface reactions and gas-grain interactions such as freeze-out and desorption. It is important not to create artificial bottlenecks in the reaction scheme by omitting important channels. The choice of reaction rate coefficients depends on factors like availability, accuracy, etc. A number of comprehensive databases of rate coefficients is available today, e.g. NSM/OHIO (Wakelam et al. 2004, 2005b), UMIST (Millar, Farquhar, \& Willacy 1997; Le Teuff et al. 2000), and Meudon (Le Bourlot et al. 1993), which collect the results from many different references, both theoretical and experimental.

An example for the importance of explicitly agreeing on the details of the computation of the reaction rate is the reaction:

$\mathrm{C}+\mathrm{H}_{2} \rightarrow \mathrm{CH}+\mathrm{H}$.

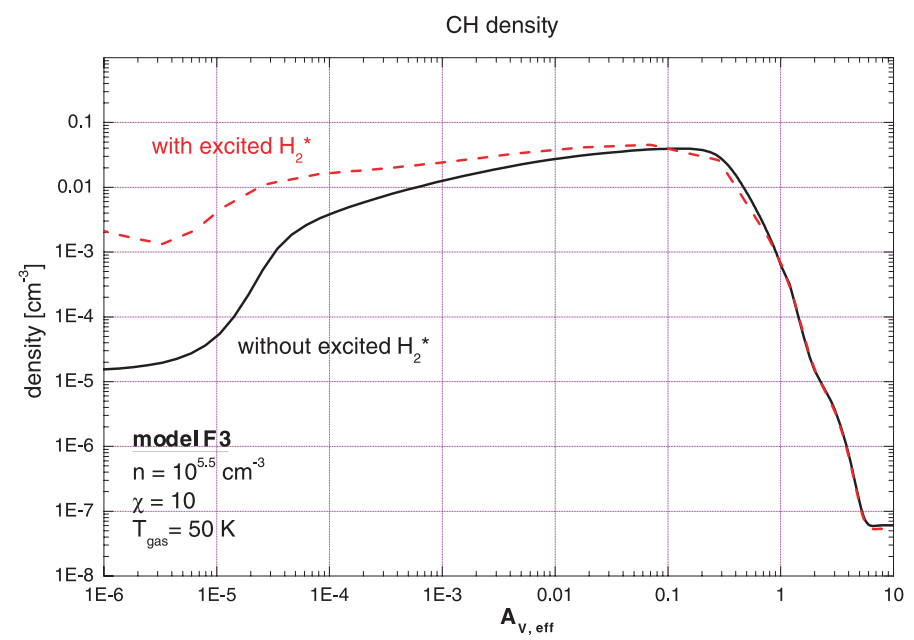

Fig. 5. Comparison between model codes with (dashed line) and without (solid line) excited molecular hydrogen, $\mathrm{H}_{2}^{*}$. The abundance profile of $\mathrm{CH}$ is plotted for both models against $A_{\mathrm{V} \text {,eff. }}$ Benchmark model F3 has a high density $\left(n=10^{5.5} \mathrm{~cm}^{-3}\right)$ and low FUV intensity $(\chi=10)$.

It has an activation energy barrier of $11700 \mathrm{~K}$ (Millar, Farquhar, \& Willacy 1997), effectively reducing the production of $\mathrm{CH}$ molecules. If we include vibrationally excited $\mathrm{H}_{2}^{*}$ into the chemical network and assume that reaction (5) has no activation energy barrier for reactions with $\mathrm{H}_{2}^{*}$ we obtain a significantly higher production rate of $\mathrm{CH}$ as shown in Figure 5. Even this approach is a rather crude assumption, but it demonstrates the importance of explicitly agreeing on how to handle the chemical calculations in model comparisons.

Another example is the formation of $\mathrm{C}$ in the dark cloud part of a PDR, i.e. at values of $A_{\mathrm{V}}>5$. A possible formation channel for atomic carbon is the dissociation of $\mathrm{CO}$ by secondary UV photons, induced by cosmic rays (Le Teuff et al. 2000). In the outer parts of the PDR the impinging FUV field dominates the dissociation of $\mathrm{CO}$, but for high $A_{\mathrm{V}}$ the FUV field is effectively shielded and CR induced UV photons become important. For $\mathrm{CO}$, this process depends on the level population of $\mathrm{CO}$, and therefore is temperature dependent (Gredel et al. 1987), however this temperature dependence is often ignored. The reaction rate given by Gredel et al. (1987) has to be corrected by a factor of $(T / 300 \mathrm{~K})^{1.17}$ effectively reducing the dissociation rate for temperatures below $300 \mathrm{~K}$ (Le Teuff et al. 2000). In Fig. 6 we plot the density profile of atomic carbon for an isothermal benchmark model with temperature $T=50 \mathrm{~K}$. The solid line represents the model result for an uncorrected photo-rate using the average reaction rate for $T=300 \mathrm{~K}$, compared to the results using the rate corrected for $T=50 \mathrm{~K}$ by $(50 / 300)^{1.17}$, given by the dashed curve.

\subsubsection{Heating and cooling}

To determine the local temperature in a cloud, the equilibrium between heating and cooling has to be calculated. The heating rates mainly depend on the $\mathrm{H}_{2}$ formation rate, the electron density, the grain size distribution, grain composition, and $\mathrm{H}_{2}$ treatment (i.e. two-line approximation vs. full ro-vib model), while the cooling rates are dominantly influenced by the abundance of the main cooling species and the dust opacity in the FIR. Table 1 gives an overview of the most important heating and cooling processes. Most of them can be modelled at different levels of detail. This choice may have a major impact 


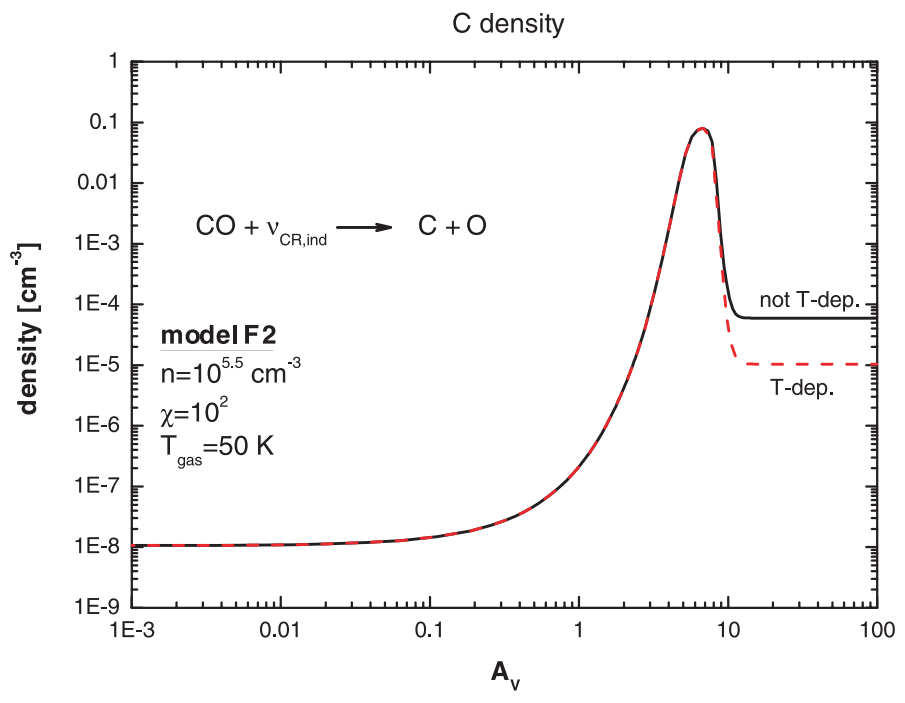

Fig. 6. The density profile of atomic carbon for the benchmark model F2 (low density, high FUV, $T=$ const. $=50 \mathrm{~K}$, as discussed in Sect. 4). The solid curve results from a constant dissociation by CR induced secondary photons (implicitely assuming $T=300 \mathrm{~K}$ ), the dashed curve shows the influence of a temperature dependent dissociation, i.e. the corresponding dissociation rate was corrected by a factor of $(T / 300 \mathrm{~K})^{1.17}$ with $T=50 \mathrm{~K}$.

Table 1. Overview over the major heating and cooling processes in PDR physics.

\begin{tabular}{ll}
\hline \hline Heating & Cooling \\
\hline photoelectric heating (dust \& PAH) & {$[\mathrm{CII}] 158 \mu \mathrm{m}$} \\
collisional de-excitation of vib. excited $\mathrm{H}_{2}$ & {$[\mathrm{OI}] 63,145 \mu \mathrm{m}$} \\
$\mathrm{H}_{2}$ dissociation & {$[\mathrm{CI}] 370,610 \mu \mathrm{m}$} \\
$\mathrm{H}_{2}$ formation & {$[\mathrm{SiII}] 35 \mu \mathrm{m}$} \\
$\mathrm{CR}$ ionization & $\mathrm{CO}, \mathrm{H}_{2} \mathrm{O}, \mathrm{OH}, \mathrm{H}_{2}$ \\
gas-grain collisions & $\mathrm{Ly} \alpha,[\mathrm{OI}],[\mathrm{FeII}]$ \\
dissipation of turbulence & gas-grain collisions \\
\hline
\end{tabular}

on the model results. One example is the influence of PAHs on the efficiency of the photoelectric heating, which results in a significantly higher temperature e.g. at the surface of the model cloud if PAHs are taken into account. Bakes \& Tielens (1994) give convenient fitting formulas for the photoelectric heating. Another important case is the collisional de-excitation of vibrationally excited $\mathrm{H}_{2}$. A detailed calculation of the level population shows that for temperatures above $800 \mathrm{~K}$ the lower transitions switch from heating to cooling. This imposes a significant influence on the net heating from $\mathrm{H}_{2}$ vibrational de-excitation. When using an approximation for the heating rate it is important to account for this cooling effect (Röllig et al. 2006). The cooling of the gas by line emission depends on the atomic and molecular constants as well as on the radiative transfer. A common approximation to the radiative transfer problem is by assuming escape probabilities for the cooling lines (de Jong et al. 1980; Stutzki 1984; Störzer et al. 1996). The excitation temperature at any point can be computed by balancing the collisional excitation and the photon escape probability. The local escape probability is obtained by integrating $\exp \left(-\tau_{v}\right)$ over $4 \pi$. In the escape probability approximation it is now assumed that the radiative interaction region is small enough so that the optical depth in each direction is produced by molecules with the same excitation temperature. Then the excitation problem becomes a local one. The [OI] $63 \mu \mathrm{m}$ line may also become very optically thick and can act both as heating and cooling contribution. Under certain benchmark conditions (low density, constant temperature $T_{\text {gas }}=50 \mathrm{~K}$ ) the $[\mathrm{OI}] 63 \mu \mathrm{m}$ line even showed weak maser behavior (see data plots at http://www .ph1. uni-koeln.de/pdr-comparison). Collisions between the gas particles and the dust grains also contribute to the total heating or cooling.

\subsubsection{Grain properties}

Many aspects of PDR physics and chemistry are connected to dust properties. We will give only a short overview of the importance of dust grains in the modeling of PDRs. Dust acts on the energy balance of the ISM by means of photoelectric heating; it influences the radiative transfer by absorption and scattering of photons, and it acts on the chemistry of the cloud via grain surface reactions, e.g. the formation of molecular hydrogen and the depletion of other species. One distinguishes three dust components: PAHs, very small grains (VSGs) and big grains (BGs).

The properties of big grains have been reviewed recently by Draine (2003, and references therein). The first indirect evidence for the presence of VSGs in the ISM was presented by Andriesse (1978) in the case of the M17 PDR. The dust grains themselves consist of amorphous silicates and carbonaceous material and may be covered with ice mantles in the denser and colder parts of the ISM. For details of the composition of grains and their extinction due to scattering and absorption see Li \& Draine (2002) and references therein.

The influence and proper treatment of electron densities together with grain ionization and recombination is still to be analyzed. Not only the charge of dust and PAHs but also the scattering properties are still in discussion (Weingartner \& Draine 2001). This heavily influences the model output, e.g. the inclusion of back-scattering significantly increases the total $\mathrm{H}_{2}$ photodissociation rate at the surface of the model cloud compared to calculations with pure forward scattering.

\subsubsection{Radiative transfer}

The radiative transfer (RT) can be split into two distinct wavelength regimes: FUV and IR/FIR. These may also be labeled as "input" and "output". FUV radiation due to ambient UV field and/or young massive stars in the neighborhood impinges on the PDR. The FUV photons are absorbed on their way deeper into the cloud, giving rise to the well known stratified chemical structure of PDRs. In general, reemission processes can be neglected in the FUV, considerably simplifying the radiative transfer problem. Traveling in only one direction, from the edge to the inside, the local mean FUV intensity can usually be calculated in a few iteration steps. In contrast to the FUV, the local FIR intensity is a function of the temperature and level populations at all positions due to absorption and reemission of FIR photons. Thus a computation needs to iterate over all spatial grid points. A common simplifying approximation is the spatial decoupling via the escape probability approximation. This allows to substitute the intensity dependence by a dependence on the relevant optical depths, ignoring the spatial variation of the source function. The calculation of emission line cooling then becomes primarily a problem of calculating the local excitation state of the particular cooling species. An overview of NLTE radiative transfer methods is given by van Zadelhoff et al. (2002). 
Table 2. List of participating codes. See Appendix for short description of the individual models.

\begin{tabular}{|c|c|}
\hline Model Name & Authors \\
\hline Cloudy & G. J. Ferland, P. van Hoof, N. P. Abel, G. Shaw (Ferland et al. 1998; Abel et al. 2005; Shaw et al. 2005) \\
\hline COSTAR & I. Kamp, F. Bertoldi, G.-J. van Zadelhoff (Kamp \& Bertoldi 2000; Kamp \& van Zadelhoff 2001) \\
\hline HTBKW & $\begin{array}{l}\text { D. Hollenbach, A. G. G. M. Tielens, M. G. Burton, M. J. Kaufman, M. G. Wolfire } \\
\text { (Tielens \& Hollenbach 1985; Kaufman et al. 1999; Wolfire et al. 2003) }\end{array}$ \\
\hline KOSMA- $\tau$ & $\begin{array}{l}\text { H. Störzer, J. Stutzki, A. Sternberg (Störzer et al. 1996), B. Köster, M. Zielinsky, U. Leuenhagen } \\
\text { Bensch et al. (2003), Röllig et al. (2006) }\end{array}$ \\
\hline Lee96mod & H.-H. Lee, E. Herbst, G. Pineau des Forêts, E. Roueff, J. Le Bourlot, O. Morata (Lee et al. 1996) \\
\hline Leiden & $\begin{array}{l}\text { J. Black, E. van Dishoeck, D. Jansen and B. Jonkheid } \\
\text { (Black \& van Dishoeck 1987; van Dishoeck \& Black 1988; Jansen et al. 1995) }\end{array}$ \\
\hline Meijerink & R. Meijerink, M. Spaans (Meijerink \& Spaans 2005) \\
\hline Meudon & J. Le Bourlot, E. Roueff, F. Le Petit (Le Petit et al. 2005, 2002; Le Bourlot et al. 1993) \\
\hline Sternberg & A. Sternberg, A. Dalgarno (Sternberg \& Dalgarno 1989, 1995; Boger \& Sternberg 2005) \\
\hline UCL_PDR & S. Viti, W.-F. Thi, T. Bell (Taylor et al. 1993; Papadopoulos et al. 2002; Bell et al. 2005) \\
\hline
\end{tabular}

\section{Description of the benchmark models}

\subsection{PDR code characteristics}

A total number of 11 model codes participated in the PDR model comparison study during and after the workshop in Leiden. Table 2 gives an overview of these codes. The codes are different in many aspects:

- finite and semi-infinite plane-parallel and spherical geometry, disk geometry;

- chemistry: steady state vs. time-dependent, different chemical reaction rates, chemical network;

- IR and FUV radiative transfer (effective or explicitly wavelength dependent), self- and mutual shielding, atomic and molecular rate coefficients;

- treatment of dust and PAHs;

- treatment of gas heating and cooling;

- range of input parameters;

- model output;

- numerical treatment, gridding, etc.

This manifold in physical, chemical and technical differences makes it difficult to directly compare results from the different codes. Thus we tried to standardize the computation of the benchmark model clouds as much as possible. This required all codes to reduce their complexity and sophistication, often beyond what their authors considered to be acceptable, considering the actual knowledge of some of the physical processes. However as the main goal of this study was to understand why and how these codes differ these simplifications are acceptable. Our aim was not to provide a realistic model of real astronomical objects. The individual strengths (and weaknesses) of each PDR code are briefly summarized in the Appendix and on the website: http://www.ph1.uni-koeln.de/pdr-comparison.

\subsection{Benchmark frame and input values}

A total of 8 different model clouds were used for the benchmark comparison. The density and FUV parameter space is covered by accounting for low and high densities and FUV fields under isothermal conditions, giving 4 different model clouds. In one set of models the complexity of the model calculations was reduced by setting the gas and dust temperatures to a given constant value (models F1-F4, "F" denoting a fixed temperature), making the results independent of the solution of the local energy balance.
Table 3. Specification of the model clouds that were computed during the benchmark. The models F1-F4 use constant gas and dust temperatures, while V1-V4 have their temperatures calculated self consistently.

\begin{tabular}{c|c}
\hline \hline F1 & F2 \\
$T=50 \mathrm{~K}$ & $T=50 \mathrm{~K}$ \\
$n=10^{3} \mathrm{~cm}^{-3}, \chi=10$ & $n=10^{3} \mathrm{~cm}^{-3}, \chi=10^{5}$ \\
\hline $\mathrm{F} 3$ & $\mathrm{~F} 4$ \\
$T=50 \mathrm{~K}$ & $T=50 \mathrm{~K}$ \\
$n=10^{5.5} \mathrm{~cm}^{-3}, \chi=10$ & $n=10^{5.5} \mathrm{~cm}^{-3}, \chi=10^{5}$ \\
\hline V1 & $\begin{array}{c}\mathrm{V} 2 \\
T=\text { variable }\end{array}$ \\
$n=10^{3} \mathrm{~cm}^{-3}, \chi=10$ & $n=10^{3} \mathrm{~cm}^{-3}, \chi=10^{5}$ \\
\hline V3 & $\begin{array}{c}\text { V } 4 \\
T=\text { variable }\end{array}$ \\
$n=10^{5.5} \mathrm{~cm}^{-3}, \chi=10$ & $n=10^{5.5} \mathrm{~cm}^{-3}, \chi=10^{5}$ \\
\hline
\end{tabular}

Table 4. Chemical content of the benchmark calculations.

\begin{tabular}{l}
\hline \hline Chemical species in the models \\
\hline $\mathrm{H}, \mathrm{H}^{+}, \mathrm{H}_{2}, \mathrm{H}_{2}^{+}, \mathrm{H}_{3}^{+}$ \\
$\mathrm{O}, \mathrm{O}^{+}, \mathrm{OH}^{+}, \mathrm{OH}, \mathrm{O}_{2}, \mathrm{O}_{2}^{+}, \mathrm{H}_{2} \mathrm{O}, \mathrm{H}_{2} \mathrm{O}^{+}, \mathrm{H}_{3} \mathrm{O}^{+}$ \\
$\mathrm{C}, \mathrm{C}^{+}, \mathrm{CH}, \mathrm{CH}^{+}, \mathrm{CH}_{2}, \mathrm{CH}_{2}^{+}, \mathrm{CH}_{3}$, \\
$\mathrm{CH}_{3}^{+}, \mathrm{CH}_{4}, \mathrm{CH}_{4}^{+}, \mathrm{CH}_{5}^{+}, \mathrm{CO}, \mathrm{CO}^{+}, \mathrm{HCO}^{+}$ \\
$\mathrm{He}^{+} \mathrm{He}^{+}, \mathrm{e}^{-}$ \\
\hline
\end{tabular}

In a second benchmark set, the thermal balance has been solved explicitly thus determining the temperature profile of the cloud (models V1-V4, "V" denoting variable temperatures). Table 3 gives an overview of the cloud parameter of all eight benchmark clouds.

\subsubsection{Benchmark chemistry}

One of the crucial steps in arriving at a useful code comparison was to agree on the use of a standardized set of chemical species and reactions to be accounted for. For the benchmark models we only included the four most abundant elements $\mathrm{H}, \mathrm{He}, \mathrm{O}$, and $\mathrm{C}$. Additionally only the species given in Table 4 are included in the chemical network calculations:

The chemical reaction rates are taken from the UMIST99 database (Le Teuff et al. 2000) together with some corrections suggested by A. Sternberg. The complete reaction rate 
file is available online (http://www.ph1.uni-koeln.de/ pdr-comparison). To reduce the overall modeling complexity, PAHs were neglected in the chemical network and were only considered for the photoelectric heating (photoelectric heating efficiency as given by Bakes \& Tielens 1994) in models V1-V4. Codes which calculate time-dependent chemistry used a suitably long time-scale in order to reach steady state (e.g. UCL_PDR used $100 \mathrm{Myr}$ ).

\subsubsection{Benchmark geometry}

All model clouds are plane-parallel, semi-infinite clouds of constant total hydrogen density $n=n(\mathrm{H})+2 n\left(\mathrm{H}_{2}\right)$. Spherical codes approximated this by assuming a very large radius for the cloud.

\subsubsection{Physical specifications}

As many model parameters as possible were agreed upon at the start of the benchmark calculations, to avoid confusion in comparing model results. To this end we set the most crucial model parameters to the following values: the value for the standard UV field was taken as $\chi=10$ and $10^{5}$ times the Draine (1978) field. For a semi-infinite plane parallel cloud the $\mathrm{CO}$ dissociation rate at the cloud surface for $\chi=10$ should equal $10^{-9} \mathrm{~s}^{-1}$, using that for optically thin conditions (for which a point is exposed to the full $4 \pi$ steradians, as opposed to $2 \pi$ at the cloud surface) the $\mathrm{CO}$ dissociation rate is $2 \times 10^{-10} \mathrm{~s}^{-1}$ in a unit Draine field. The cosmic ray $\mathrm{H}$ ionization rate is assumed to be $\zeta=5 \times 10^{-17} \mathrm{~s}^{-1}$ and the visual extinction $A_{\mathrm{V}}=6.289 \times 10^{-22} N_{\mathrm{H} \text {,tot }}$. If the codes do not explicitly calculate the $\mathrm{H}_{2}$ photo-dissociation rates (by summing over oscillator strengths etc.) we assume that the unattenuated $\mathrm{H}_{2}$ photo-dissociation rate in a unit Draine field is equal to $5.18 \times 10^{-11} \mathrm{~s}^{-1}$, so that at the surface of a semi-infinite cloud for 10 times the Draine field the $\mathrm{H}_{2}$ dissociation rate is $2.59 \times$ $10^{-10} \mathrm{~s}^{-1}$ (numerical values from Sternberg. See Sect. 5.1 for a discussion on $\mathrm{H}_{2}$ dissociation rates). For the dust attenuation factor in the $\mathrm{H}_{2}$ dissociation rate we assumed $\exp \left(-k A_{\mathrm{V}}\right)$ if not treated explicitly wavelength dependent. The value $k=3.02$ is representative for the effective opacity in the 912-1120 A range (for a specific value of $R_{\mathrm{V}} \approx 3$ ). We use a very simple $\mathrm{H}_{2}$ formation rate coefficient $R=3 \times 10^{-18} T^{1 / 2}=2.121 \times^{-17} \mathrm{~cm}^{3} \mathrm{~s}^{-1}$ (Sternberg \& Dalgarno 1995) at $T=50 \mathrm{~K}$, assuming that every atom that hits a grain sticks and reacts to $\mathrm{H}_{2}$. A summary of the most important model parameters is given in Table 5 .

\section{Results}

In the following section we give a short overview of the up to date results of the PDR model comparison. The names of the model codes are printed in typewriter font (e.g. COSTAR). We will refer to the two stages of the benchmarking results by pre- and post-benchmark, denoting the model results at the beginning of the comparison and at its end respectively. All pre- and post-benchmark results are posted at http://www.ph1.uni-koeln.de/pdr-comparison. One model from the initial 12 participating model was left out in the post-benchmark plots because the authors could not attend the workshop. In addition, the KOSMA- $\tau$ models (Röllig et al. 2006) and the models by Bensch, which participated in the comparison as seperate codes, have been merged to a single set (labeled KOSMA- $\tau$ ) as they are variants on of the same basic model which do not differ for the given benchmarking parameter set, and consequently give identical results. To demonstrate the impact of the benchmark effort on the results of the
Table 5. Overview of the most important model parameter. All abundances are given w.r.t. total $\mathrm{H}$ abundance.

\begin{tabular}{lll}
\hline \hline & \multicolumn{2}{c}{ Model Parameters } \\
\hline$A_{\mathrm{He}}$ & 0.1 & Elemental He abundance \\
$A_{\mathrm{O}}$ & $3 \times 10^{-4}$ & Elemental O abundance \\
$A_{\mathrm{C}}$ & $1 \times 10^{-4}$ & Elemental C abundance \\
$\zeta_{\mathrm{CR}}$ & $5 \times 10^{-17} \mathrm{~s}^{-1}$ & CR ionization rate \\
$A_{\mathrm{V}}$ & $6.289 \times 10^{-22} N_{\mathrm{H}_{\text {total }}}$ & Visual extinction \\
$\tau_{\mathrm{UV}}$ & $3.02 A_{v}$ & FUV dust attenuation \\
$v_{\mathrm{b}}$ & $1 \mathrm{~km} \mathrm{~s}^{-1}$ & Doppler width \\
$D_{\mathrm{H}_{2}}$ & $5 \times 10^{-18} \cdot \frac{\chi}{10} \mathrm{~s}^{-1}$ & $\mathrm{H}_{2}$ dissociation rate \\
$R$ & $3 \times 10^{-18} T^{1 / 2} \mathrm{~cm}^{3} \mathrm{~s}^{-1}$ & $\mathrm{H}_{2}$ formation rate \\
$T_{\text {gas,fix }}$ & $50 \mathrm{~K}$ & Gas temperature (for F1-F4) \\
$T_{\text {dust,fix }}$ & $20 \mathrm{~K}$ & Dust temperature (for F1-F4) \\
$n$ & $10^{3}, 10^{5.5} \mathrm{~cm}^{-3}$ & Total density \\
$\chi$ & $10,10^{5}$ & FUV intensity w.r.t. \\
& & Draine (1978) field \\
& & (i.e. $\chi=1.71 G_{0}$ ) \\
\hline
\end{tabular}

participating $\mathrm{PDR}$ codes we plot the well known $\mathrm{C} / \mathrm{C}^{+} / \mathrm{CO}$ transition for a typical PDR environment before and after the changes identified as necessary during the benchmark in Fig. 7. The photo-dissociation of carbon monoxide is thought to be well understood for almost 20 years (van Dishoeck \& Black 1988). Nevertheless we see a significant scatter for the densities of $\mathrm{C}^{+}, \mathrm{C}$, and $\mathrm{CO}$ in the top plot of Fig. 7. The scatter in the pre-benchmark rates is significant. Most deviations could be assigned to either bugs in the pre-benchmark codes, misunderstandings, or to incorrect geometrical factors (e.g. $2 \pi$ vs. $4 \pi$ ). This emphasizes the importance of this comparative study to establish a uniform understanding about how to calculate even these basic figures. Despite the considerable current interest because of, e.g. SPITZER results, we do not give the post-benchmark predictions for the $\mathrm{H}_{2}$ mid-IR and near IR lines (or the corresponding Boltzmann diagram). Only a small fraction of the participating codes is able to compute the detailed $\mathrm{H}_{2}$ population and emission, and the focus of this analysis is the comparison between the benchmark codes.

\subsection{Models with constant temperature F1-F4}

The benchmark models F1 to F4 were calculated for a fixed gas temperature of $50 \mathrm{~K}$. Thus, neglecting any numerical issues, all differences in the chemical structure of the cloud are due to the different photo-rates, or non-standard chemistry. Some PDR codes used slightly different chemical networks. The code Sternberg uses the standard chemistry with the addition of vibrational excited hydrogen and a smaller $\mathrm{H}-\mathrm{H}_{2}$ formation network. The results by Cloudy were obtained with two different chemical setups: The pre-benchmark chemistry had the chemical network of Tielens \& Hollenbach (1985). The post benchmark results use the corrected UMIST database. Cloudy also used a different set of radiative recombination coefficients for the pre-benchmark calculations which were the major source for their different results (Abel et al. 2005). The carbon photoionization and radiative recombination rates are very sensitive to radiative transfer and hence to dust properties. The dust properties in Cloudy are different from what is implicitly assumed in the UMIST fits. Cloudy's post-benchmark results are achieved after switching to the benchmark specifications. After 

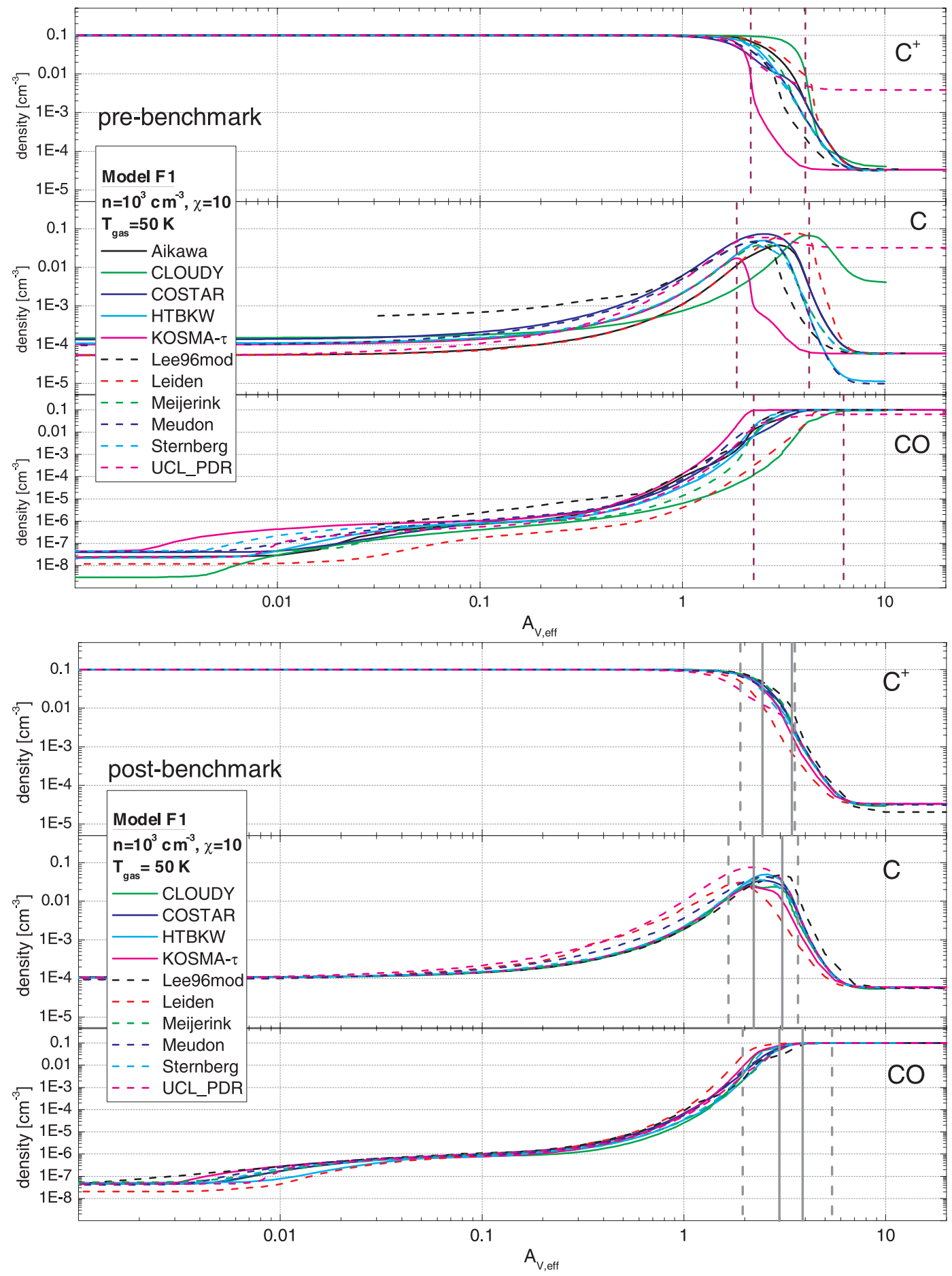

Fig. 7. Model F1 ( $\left.n=10^{3} \mathrm{~cm}^{-3}, \chi=10\right)$ : comparison between the density profiles of $\mathrm{C}^{+}$(top), $\mathrm{C}$ (middle), and CO (bottom) before (top) and after (bottom) the comparison study. The vertical lines indicate the code dependent scatter. For $\mathrm{C}$ and $\mathrm{CO}$ they indicate the depths at which the maximum density is reached, while for $\mathrm{C}^{+}$they indicate the depths at which the density dropped by a factor of 10 . Dashed lines indicate pre-benchmark results, while solid lines are post-benchmark.

the switch they agree very well with the other codes. In Fig. 7 we present the pre- and post-benchmark results for the main carbon bearing species $\mathrm{C}^{+}, \mathrm{C}$, and $\mathrm{CO}$. To emphasize the pre-to-post changes we added several vertical marker lines to the plots. For $\mathrm{C}$ and $\mathrm{CO}$ they indicate the depths at which the maximum density is reached, while for $\mathrm{C}^{+}$they indicate the depths at which the density has dropped by a factor of 10 . Dashed lines indicate pre-benchmark results, while solid lines are post-benchmark. In the pre-benchmark results the code dependent scatter for these depths is $\Delta A_{\mathrm{V}, \mathrm{eff}} \approx 2-4$ and drops to $\Delta A_{\mathrm{V}, \mathrm{eff}} \approx 1$ in the postbenchmark results.

In the post-benchmark results, the Leiden and UCL_PDR models show a slightly different behavior. The predicted peak depth of $\mathrm{C}$ is somewhat smaller than for the other codes. The peak C density of UCL_PDR is roughly $50 \%$ higher than in the other codes. A comparison with the photo-ionization of $\mathrm{C}$ shown in Fig. 8 confirms that a slightly stronger shielding for the ionization of $\mathrm{C}$ is the reason for the different behavior of $\mathrm{C}$ and $\mathrm{C}^{+}$. 


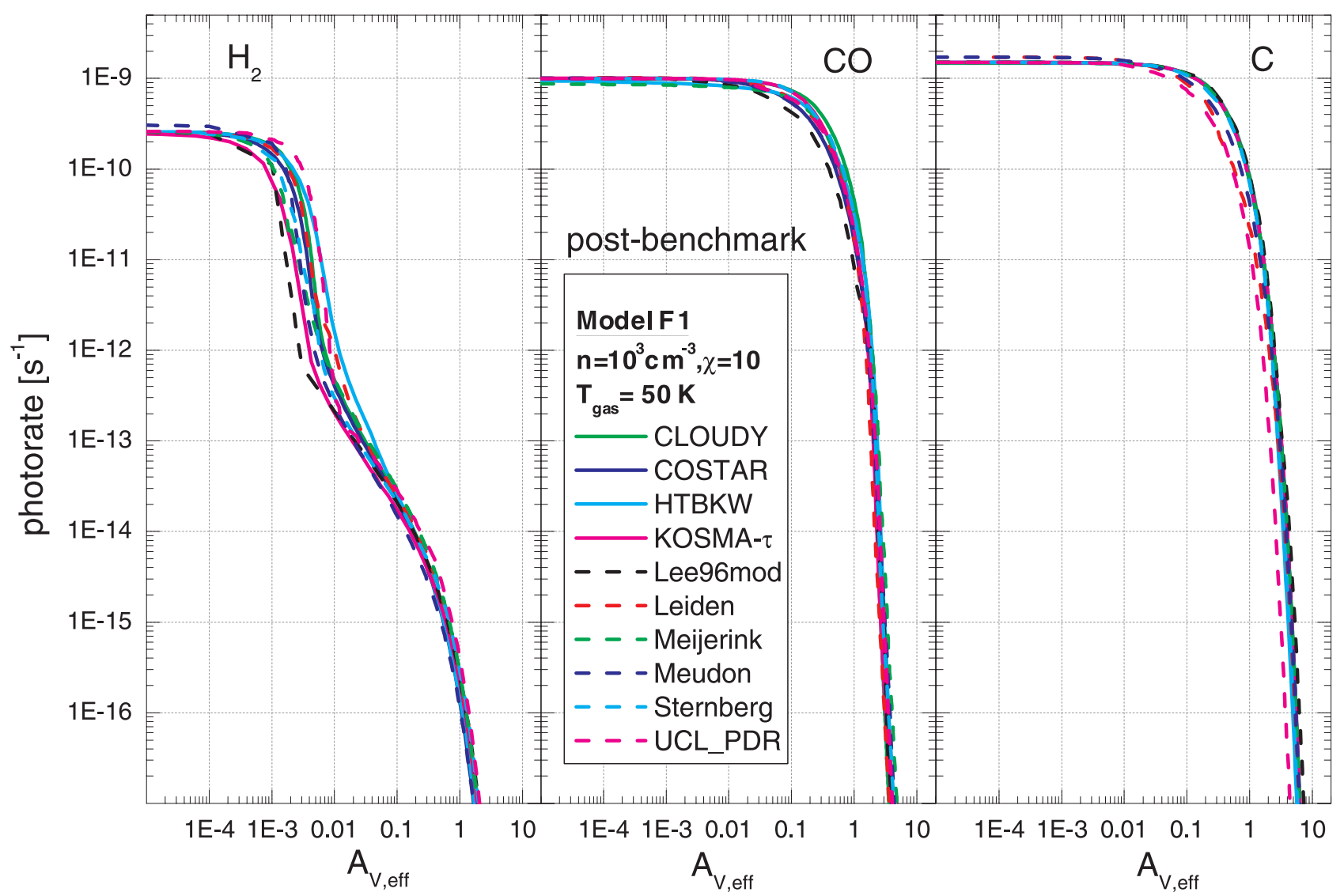

Fig. 8. Model F1 $\left(n=10^{3} \mathrm{~cm}^{-3}, \chi=10\right)$ : the photo-dissociation rates of $\mathrm{H}_{2}$ (left column), of $\mathrm{CO}$ (middle column) and the photo-ionization rate of $\mathrm{C}$ (right column) after the comparison study.

The dark cloud densities for $\mathrm{C}^{+}, \mathrm{C}$, and $\mathrm{CO}$ agree very well, except for a somewhat smaller $\mathrm{C}^{+}$density in the Lee96mod results.

In Fig. 8 we plot the post-benchmark photo-rates for dissociation of $\mathrm{H}_{2}$ (left column) and $\mathrm{CO}$ (middle column) and for the ionization of C (right column), computed for model F1. Even for this simple model there are some significant differences between the models in the various rates. In the pre-benchmark results, several codes calculated different photo-rates at the edge of the model cloud, i.e. for very low values of $A_{\mathrm{V} \text {,eff }}$. Some codes calculated surface photo-dissociation rates between $4-5 \times 10^{-10} \mathrm{~s}^{-1}$ compared to the expected value of $2.59 \times 10^{-10} \mathrm{~s}^{-1}$. Most of these deviations were due to exposure to the full $4 \pi$ steradians FUV field instead the correct $2 \pi$, but also due to different effects, like the FUV photon back-scattering in the Meudon results. The pre-benchmark rates of KOSMA- $\tau$ were shifted toward slightly lower values of $A_{\mathrm{V}}$ because of an incorrect scaling between $A_{\mathrm{V}}$ and $A_{\mathrm{V} \text {,eff }}$ and an incorrect calculation of the angular averaged photo-rate (the model features a spherical geometry with isotropic FUV illumination). The post-benchmark results (Fig. 8) show that most deviations have been corrected. The remaining offset for the Meudon result is due to the consideration of backscattered FUV photons, increasing the local mean FUV intensity. The pre- to post-benchmark changes for the photorates of $\mathrm{CO}$ and $\mathrm{C}$ are even more convincing (see online archive). The post-benchmark results are in very good agreement except for some minor difference, e.g. UCL_PDR's photo-ionization rate of $\mathrm{C}$ showing some deviation from the main field.

The depth-dependence of the $\mathrm{H}_{2}$ photo-dissociation rate is reflected in the structure of the $\mathrm{H}-\mathrm{H}_{2}$ transition zone. Figure 9 shows the densities of atomic and molecular hydrogen after

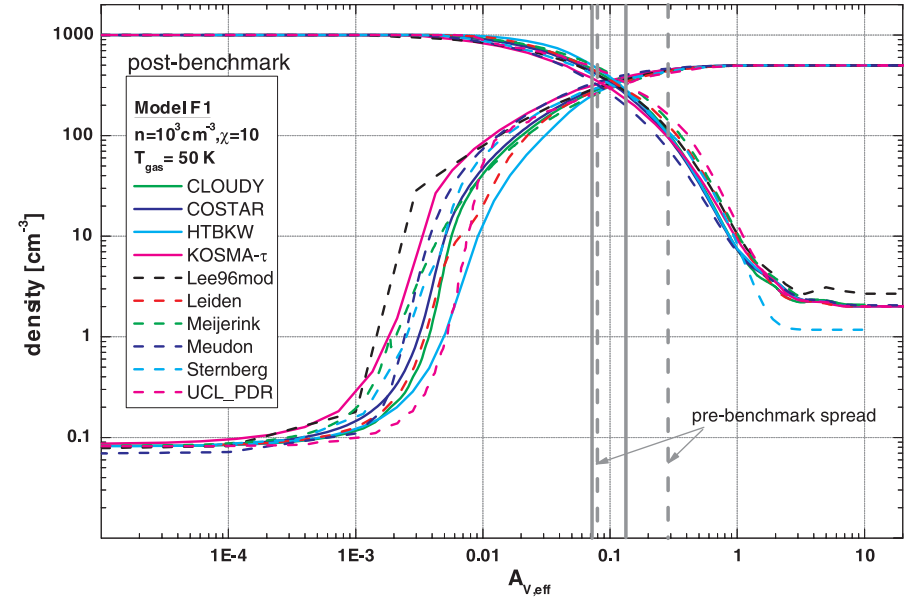

Fig. 9. Model $\mathrm{F} 1\left(n=10^{3} \mathrm{~cm}^{-3}, \chi=10\right)$ The $\mathrm{H}-\mathrm{H}_{2}$ transition zone after the comparison study. Plotted is the number density of atomic and molecular hydrogen as a function of $A_{\mathrm{V}, \text { eff }}$. The vertical lines denote the range of the predicted transition depths for pre- and post-benchmark results (dashed and solid lines respectively).

the benchmark. The vertical lines denote the minimum and maximum transition depths before (dashed) and after the benchmark (solid). In the pre-benchmark results the predicted transition depth ranges from $0.08 A_{\mathrm{V} \text {,eff }}$ to $0.29 A_{\mathrm{V} \text {,eff. In the }}$ post-benchmark results this scatter is reduced by more than a factor of 3 . Sternberg gives a slightly smaller $\mathrm{H}$ density in the dark cloud part. In this code, cosmic ray (CR) destruction and grain surface formation are the only reactions considered in 


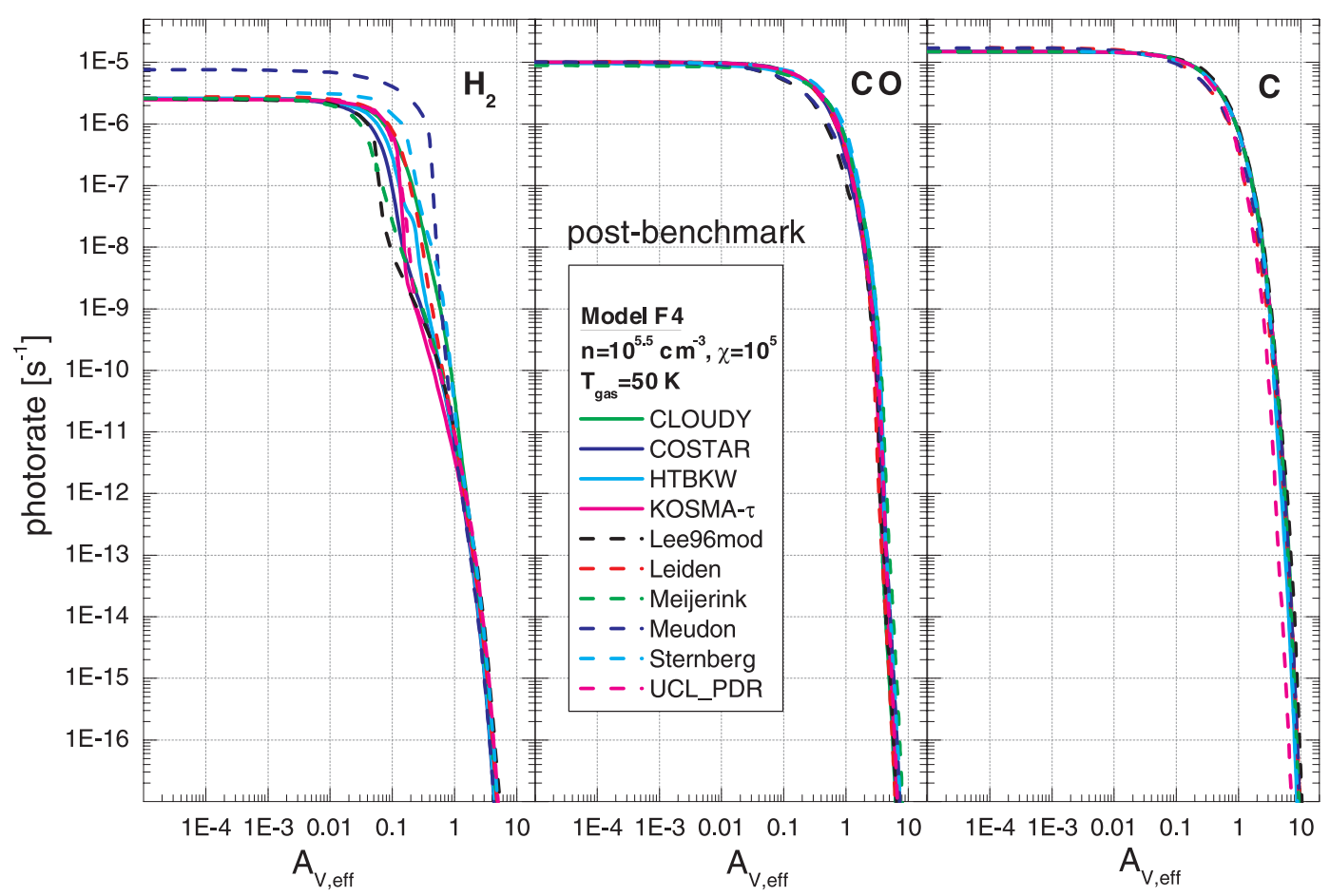

Fig. 10. Model F4 $\left(n=10^{5.5} \mathrm{~cm}^{-3}, \chi=10^{5}\right)$ : the post-benchmark photo-dissociation rates of $\mathrm{H}_{2}$ (left column), of CO (middle column) and the photo-ionization rate of $\mathrm{C}$ (right column) (upper plot).

the calculation of the $\mathrm{H}_{2}$ density. The other codes use additional reactions. The reactions:

$$
\begin{aligned}
& \mathrm{H}_{2}^{+}+\mathrm{H}_{2} \rightarrow \mathrm{H}_{3}^{+}+\mathrm{H} \quad\left(k=2.08 \times 10^{-9} \mathrm{~cm}^{3} \mathrm{~s}^{-1}\right) \\
& \mathrm{H}_{2}+\mathrm{CH}_{2}^{+} \rightarrow \mathrm{CH}_{3}^{+}+\mathrm{H} \quad\left(k=1.6 \times 10^{-9} \mathrm{~cm}^{3} \mathrm{~s}^{-1}\right)
\end{aligned}
$$

contribute to the total $\mathrm{H}$ density at high $A_{\mathrm{V}, \text { eff. }}$ This results in a somewhat higher $\mathrm{H}$ density as shown in Fig. 9. The Meudon model gives a slightly smaller $\mathrm{H}_{2}$ density at the edge of the cloud than the other codes. This is due to the already mentioned higher photo-dissociation rate of molecular hydrogen applied in their calculations.

The model F1 may represent a typical translucent cloud PDR, e.g., the line of sight toward HD 147889 in Ophiuchus (Liseau et al. 1999). The low density and FUV intensity conditions emphasize some effects that would be hard to notice otherwise. This includes purely numerical issues like gridding and interpolation/extrapolation of shielding rates. These differences explain why the various codes still show some post-benchmark scatter. We relate differences in the predicted abundances to the corresponding rates for ionization and dissociation.

Since most of the codes use the same chemical network and apply the same temperature, the major source for remaining deviations should be related to the FUV radiative transfer. To study this we present some results of benchmark model F4 featuring a density $n=10^{5.5} \mathrm{~cm}^{-3}$ and a FUV intensity $\chi=10^{5}$, in order to enhance any RT related differences and discuss them in more detail. Figure 10 shows the post-benchmark photo-rates for the model F4. The higher unshielded $\mathrm{H}_{2}$ photo-rate in the Meudon results, already visible in model F1 (Fig. 8) is now significantly enhanced due to the increased FUV flux. Meudon, as well as Cloudy, Leiden and Sternberg, treat the hydrogen molecule by calculating the local level population and determining the photo-dissociation rate by integrating each absorption line over the absorption cross section and summing over all absorption lines. Meudon, Cloudy, and Leiden integrate the line profile over the attenuated spectrum, in order to account for line overlap effects, while Sternberg treats each line seperately, neglecting line overlap. Most other codes just assume that the photodissociation scales with the incident radiation field, neglecting any influence from varying $\mathrm{H}_{2}$ level populations. One reason for the different $\mathrm{H}_{2}$ photo-rate is a different local mean FUV intensity, caused by backscattered photons. However, this should only account for approximately $10 \%$ of the increased dissociation rate. The remaining differences are due to different treatment of $\mathrm{H}_{2}$. Either they use different equations, e.g. full ro-vib resolution in Meudon and Sternberg vs. only vib. population in KOSMA- $\tau$, or they apply different molecular data. Sternberg uses data from Sternberg \& Dalgarno (1989); Sternberg \& Neufeld (1999). Meudon uses collisional data from Flower (1997, 1998); Flower \& Roueff (1999) and associated papers, and radiative data from Abgrall et al. (2000), including dissociation efficiencies. These different data sets result in:

1. excited rotational states are much more populated in Meudon's results than in Sternberg;

2. dissociation from an excited rotational level increases much faster with J in Meudon's data.

Both effects lead to dissociation probabilities that differ by 2-3 in case of Model F4. Due to the structure of the code these features could not be turned off in Meudon results.

The photo-rates for $\mathrm{CO}$ and $\mathrm{C}$ are in very good accord, but we notice a considerable spread in the shielding behavior of the hydrogen photo-rate. This spread is due to the particular implementation of $\mathrm{H}_{2}$ shielding native to every code, by either using tabulated shielding functions or explicitly calculating the total cross section at each wavelength. The different photo-rates directly cause a different $\mathrm{H}-\mathrm{H}_{2}$ transition profile, shown in the top panel of Fig. 11. The low molecular hydrogen densities in the Meudon and Cloudy models are again due to the higher $\mathrm{H}_{2}$ 

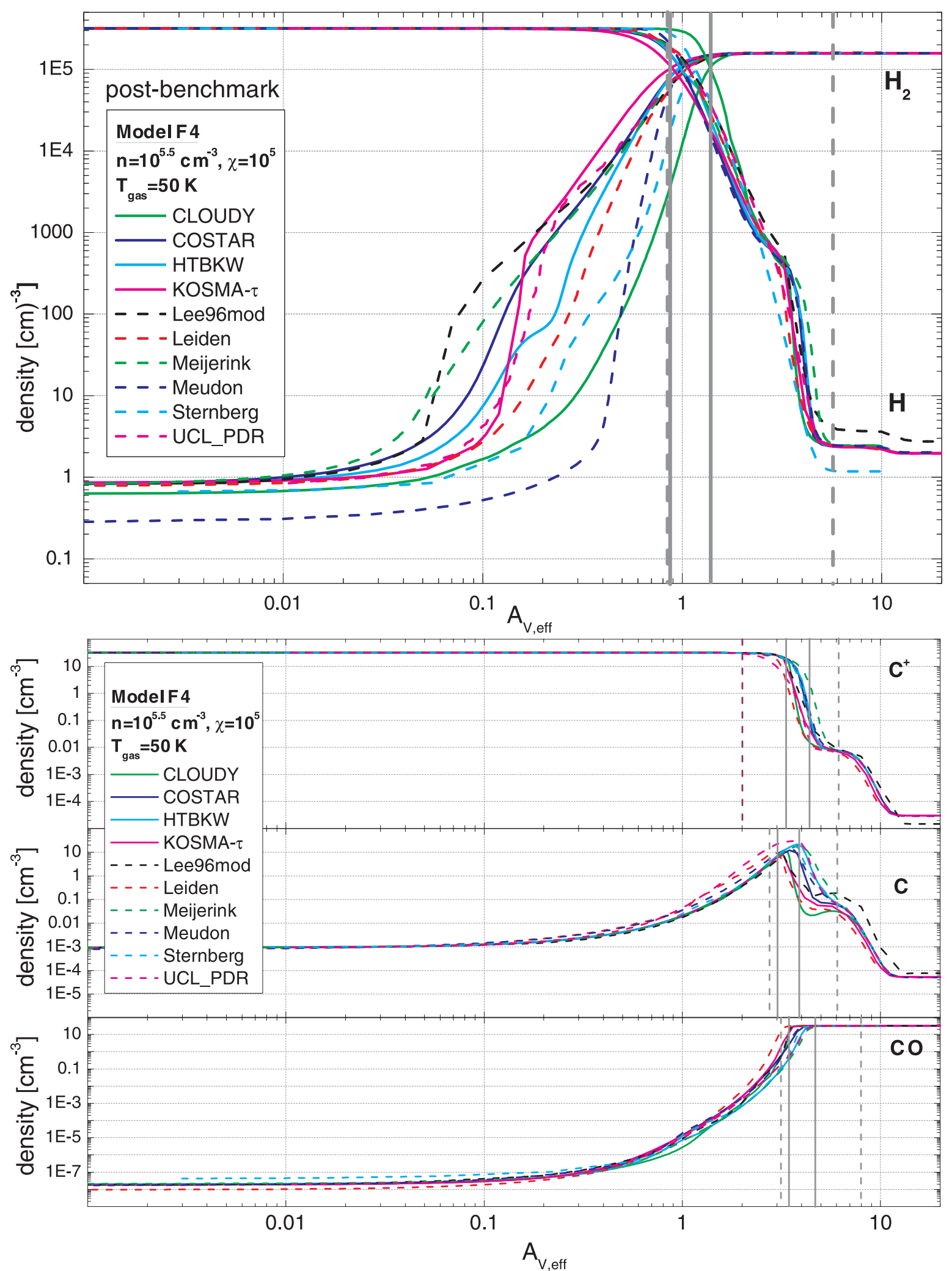

Fig. 11. Model F4 $\left(n=10^{5.5} \mathrm{~cm}^{-3}, \chi=10^{5}\right)$ : The upper panel shows the post-benchmark results for the $\mathrm{H}$ and $\mathrm{H}_{2}$ densities. The lower panel shows the post-benchmark density profiles of $\mathrm{C}^{+}, \mathrm{C}$, and $\mathrm{CO}$. The vertical gray lines in both panels indicate the pre-to-post changes.

photo-dissociation rate. Sternberg's slightly lower $\mathrm{H}_{2}$ abundance at the edge of the cloud is consistent with the marginally higher, unshielded $\mathrm{H}_{2}$ photo-dissociation rate, seen in the top plot in Fig. 10. The Meijerink code shows the earliest drop in the photo-rate, reflected by the corresponding increase in the
$\mathrm{H}_{2}$ density. The qualitatively different $\mathrm{H}_{2}$ profile in KOSMA $-\tau$ is most likely due to the spherical geometry in the code. Again Sternberg produces slightly smaller $\mathrm{H}$ densities for high values of $A_{\mathrm{V} \text {,eff }}$. Since Sternberg does not consider additional reactions for the $\mathrm{H} / \mathrm{H}_{2}$ balance its $\mathrm{H}$ density profile is the only one 
not showing the slight kink at $A_{\mathrm{V} \text {,eff }} \approx 2 \ldots 3$. These deviations do not significantly change the total column density of hydrogen. Hence the impact on any comparison with observational findings is small. Nevertheless one would expect that under the standardized benchmark conditions all codes produce very similar results, yet we note a considerable spread in hydrogen abundances for $A_{\mathrm{V} \text {,eff }}>2$. This again emphasizes how complex and difficult the numerical modeling of PDRs is. The bottom panel in Fig. 11 shows the density profiles of $\mathrm{C}^{+}, \mathrm{C}$, and $\mathrm{CO}$. Here, the different codes are in good agreement. The largest spread is visible for the $\mathrm{C}$ density between $A_{\mathrm{V} \text {,eff }} \approx 3 \ldots 6$. The results for $\mathrm{C}^{+}$and $\mathrm{CO}$ differ less. Lee96mod's results for $\mathrm{C}^{+}$and $\mathrm{C}$ show a small offset for $A_{\mathrm{V} \text {,eff }}>6$. They produce slightly higher $\mathrm{C}$ abundances and lower $\mathrm{C}^{+}$abundances in the dark cloud part. The different codes agree very well in the predicted depth where most carbon is locked up in $\mathrm{CO}\left(A_{\mathrm{V}, \mathrm{eff}} \approx 3.5 \ldots 4.5\right)$. This range improved considerably compared to the pre-benchmark predictions of $A_{\mathrm{V}, \mathrm{eff}} \approx 3 \ldots 8$.

The results from models F1-F4 clearly demonstrate the importance of the PDR code benchmarking effort. The prebenchmark results show a significant code-dependent scatter. Although many of these deviations have been removed during the benchmark activity, we did not achieve identical results with different codes. Many uncertainties remained even in the isothermal case, raising the need for a deeper follow up study.

\subsection{Models with variable temperature V1-V4}

In the benchmark models V1-V4 the various codes were required to also solve the energy balance equations in order to derive the temperature structure of the model clouds. This of course introduces an additional source of variation between the codes. The chemical rate equations strongly depend on the local temperature, hence we expect a strong correlation between temperature differences and different chemical profiles of the model codes. As a consequence of a differing density profile of e.g. $\mathrm{CO}$ and $\mathrm{H}_{2}$ we also expect different shielding signatures. We will restrict ourselves to just a few exemplary non-isothermal results because a full analysis of the important non-isothermal models goes beyond the scope of this paper. To demonstrate the influence of a strong FUV irradiation we show results for the benchmark model V2 with $n=10^{3} \mathrm{~cm}^{-3}$, and $\chi=10^{5}$ in Figs. 12-16. The detailed treatment of the various heating and cooling processes differs significantly from code to code. The only initial benchmark requirements was to treat the photoelectric (PE) heating according to Bakes \& Tielens (1994). On one hand, this turned out to be not strict enough to achieve a sufficient agreement for the gas temperatures, on the other hand it was already too strict to be easily implemented for some codes, like Cloudy, which calculates the PE heating self-consistently from a given dust composition. This demonstrates that there are limits to the degree of standardization. The calculation of the dust temperature was not standardized and varies from code to code. Since Lee96mod only accounts for constant temperatures, their model is not shown in the following plots. We only plot the final, post-benchmark status.

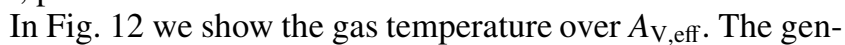
eral temperature profile is reproduced by all codes. Even so we note considerable differences between different codes. The derived temperatures at the surface vary between 1600 and $2500 \mathrm{~K}$. For low values of $A_{\mathrm{V} \text {,eff }}$ the heating is dominated by PE heating due to the high FUV irradiation, and the main cooling is provided by [OI] and [CII] emission. It is interesting, that the dominant cooling line is the [OI] $63 \mu \mathrm{m}$ line (cf. Fig. 16, left plot),

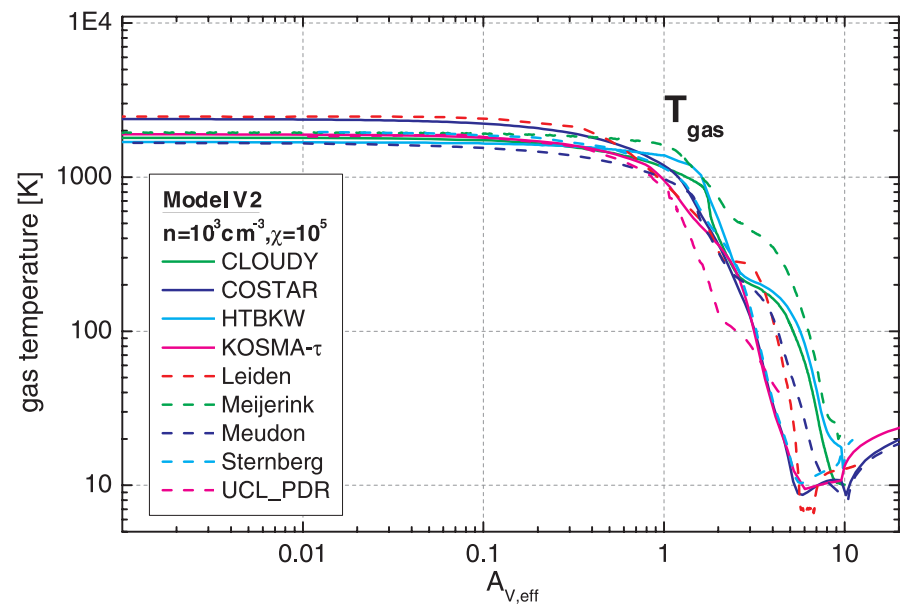

Fig. 12. Model V2 $\left(n=10^{3} \mathrm{~cm}^{-3}, \chi=10^{5}\right)$ : the plot shows the postbenchmark results for the gas temperature.

although its critical density is two orders of magnitude higher than the local density $\left(n_{\mathrm{cr}} \approx 5 \times 10^{5} \mathrm{~cm}^{-3}\right)$. The highest surface temperature is calculated by Leiden, while Meudon computes the lowest temperature. The bulk of models gives surface temperatures near $1900 \mathrm{~K}$. All models qualitatively reproduce the temperature behavior at higher values of $A_{\mathrm{V} \text {,eff }}$ and show a minimum temperature of $10 \mathrm{~K}$ between $A_{\mathrm{V} \text {,eff }} \approx 5 \ldots 10$, followed by a subsequent rise in temperature. The only relevant heating contribution at $A_{\mathrm{V} \text {,eff }}>5$ comes from cosmic ray heating, which hardly depends on $A_{\mathrm{V} \text {,eff }}$. At $A_{\mathrm{V} \text {,eff }}>4$, the dominant cooling is by $[\mathrm{CI}]$ fine structure emission. This is a very efficient cooling process and the temperature reaches its minimum. At $A_{\mathrm{V}, \text { eff }}=10$ the atomic carbon density rapidly drops and $\mathrm{CO}$ cooling starts to exceed the fine structure cooling (cf. abundance profiles in Fig. 14). However, cooling by $\mathrm{CO}$ line emission is much less efficient, especially at these low total densities, and thus the temperature increases again.

For the bulk of the cloud the heating contribution by $\mathrm{H}_{2}$ vibrational deexcitation is negligible compared to photoelectric heating. Only Mei jerink and Leiden predict comparable contributions from both processes. Unfortunately, the exact treatment of this process was not standardized and depends very much on the detailed implementation (e.g. the two-level approximation from Burton et al. (1990) or Röllig et al. (2006) vs. the solution of the full $\mathrm{H}_{2}$ problem like in Meudon, Cloudy, and Sternberg). Generally the heating by $\mathrm{H}_{2}$ vibrational deexcitation depends on the local density and the local mean FUV intensity, and should thus decrease at large values of $A_{\mathrm{V} \text {,eff }}$ and dominate the heating for denser clouds.

At $A_{\mathrm{V}, \text { eff }} \approx 2 \ldots 3$ we note a flattening of the temperature curve in many models, followed by a steeper decline somewhat deeper inside the cloud. This is not the case for HTBKW, KOSMA- $\tau$, and Sternberg. The reason for this difference is the [OI] $63 \mu \mathrm{m}$ cooling, showing a steeper decline for the three codes (Fig. 16, left plot). For very large depths, KOSMA- $\tau$ produces slightly higher gas temperatures. This is due to the larger dust temperature and the strongest $\mathrm{H}_{2}$ vibrational deexcitation heating at $A_{\mathrm{V}, \text { eff }}>10$.

In Fig. 13 we plot the photodissociation rate of $\mathrm{H}_{2}$ (top left), the photoioniozation rate of $\mathrm{C}$ (top right), and the density of $\mathrm{H}$ and $\mathrm{H}_{2}$ over $A_{\mathrm{V} \text {,eff }}$ (bottom). Meudon's unshielded dissociation rate is by a factor three larger than the median of $2.6 \times 10^{-6} \mathrm{~s}^{-1}$, and the Sternberg value of $3.8 \times 10^{-6} \mathrm{~s}^{-1}$ is slightly larger for the same reason as discussed in Sect. 5.1. The depth dependent 
shielding shows good agreement between all codes, with slight variations. The different model geometry of KOSMA $-\tau$ is reflected in the slightly stronger shielding. Leiden has the weakest shielding. Like some of the other codes (see Appendix), they account for the detailed $\mathrm{H}_{2}$ problem when calculating the photodissociation rate, instead of applying tabulated shielding rates. Yet these differences are small, since we are in a parameter regime $(\chi / n=100)$, where the main shielding is dominated by dust rather than by self shielding (Draine \& Bertoldi 1996). The density profiles of $\mathrm{H}$ and $\mathrm{H}_{2}$ are in good agreement. The stronger photodissociation in Meudon is reflected in their smaller $\mathrm{H}_{2}$ density at the surface. All other $\mathrm{H}_{2}$ densities correspond well to their dissociation rates except for Cloudy, which has a lower density at the surface without a corresponding photodissociation rate. This is a temperature effect. Cloudy computes relatively low surface temperatures which lead to slightly lower $\mathrm{H}$ densities at the surface. The central densities are also in good accord. The different $\mathrm{H}$ densities reflect the corresponding temperature profiles from Fig. 12.

The photoionization rate of $\mathrm{C}$ is given in the top right plot in Fig. 13. All models are in good agreement at the surface of the cloud. Meudon and UCL_PDR drop slightly earlier than the bulk of the results. This is also reflected in their $\mathrm{C}$ density profiles in Fig. 14 (top right) which incline slightly earlier. Deep inside the cloud Sternberg and HTBKW show a steeper decline compared to the other codes. The agreement for the $\mathrm{C}^{+}$profile is also very good. At $A_{\mathrm{V} \text {,eff }}=5$ the densities drop by a factor of 10 and remain constant until they drop at $A_{\mathrm{V} \text {,eff }} \approx 10$. This plateau is caused by the increase in $\mathrm{C}$ density, compensating the FUV attenuating. Leiden's results show some deviations for $A_{\mathrm{V}, \text { eff }}>10$. Their $\mathrm{C}$ density remains higher throughout the center, causing a slightly different carbon and oxygen chemistry at $A_{\mathrm{V}, \text { eff }}>10$. The calculated $\mathrm{O}$ and $\mathrm{O}_{2}$ densities are given in Fig. 14 (bottom, right). The dark cloud densities are in very good agreement among the models, with some deviations in the Leiden values. The $\mathrm{O}_{2}$ profiles show some variations between $A_{\mathrm{V}, \mathrm{eff}} \approx 1$ and 10 but these are minor deviations especially taking the fact that the densities vary over 14 orders of magnitude from the outside to the center of the cloud! The differences in $\mathrm{O}_{2}$ are also reflected in the CO plot (Fig. 14, bottom left). All codes produce very similar density profiles and dark cloud values. Leiden gives a smaller CO density beyond $A_{\mathrm{V} \text {,eff }}=10$.

In Fig. 15 we plot the total surface brightnesses of the main fine-structure cooling lines for the V2 model: [CII] $158 \mu \mathrm{m}$, [OI] 63, and $146 \mu \mathrm{m}$, and [CI] 610 and $370 \mu \mathrm{m}$. For the spherical PDR models, the surface brightness averaged over the projected area of the clump is shown. The surface brightness of these fine-structure lines is smaller by typically a few $10 \%$, if calculated along a pencil-beam toward the clump center as they are enhanced in the outer cloud layers. Compared with the prebenchmark results, the spread in $T_{\mathrm{B}}$ has been decreased significantly from almost 3 orders of magnitude to a factor of 3-5 for $[\mathrm{CII}]$ and $[\mathrm{OI}]$. To explain the differences in Fig. 15 we plot in Fig. 16 the radial profiles of the local emissivities of [OI] $63 \mu \mathrm{m}$ and [CI] $310 \mu \mathrm{m}$. Leiden gives the highest [OI] brightnesses and also computes higher local [OI] $63 \mu \mathrm{m}$ emissivities for small values of $A_{\mathrm{V} \text {,eff, }}$ shown in Fig. 16. COSTAR, with very similar results for the density profile and comparable gas temperatures, gives much smaller emissivities. The reason for these deviations is still unclear. The model dependent spread in surface brightnesses is largest for the [CI] lines. HTBKW computes 10 times higher line intensities for the [CI] $370 \mu \mathrm{m}$ transition than Sternberg. This can be explained as follows.
Both codes show almost identical column densities and abundance profiles of $\mathrm{C}^{0}$, yet the local emissivities are very different between $A_{\mathrm{V} \text {,eff }}=4 \ldots 9$ (Fig. 16). Sternberg, together with some other codes, compute a local minimum for the cooling at $A_{\mathrm{V}, \mathrm{eff}} \approx 6$, while the HTBKW, Cloudy, Meijerink, and Meudon models peak at the same depth. This can be explained as a pure temperature effect, since the codes showing a $[\mathrm{CI}]$ peak compute a significantly higher temperature at $A_{\mathrm{V}, \text { eff }}=6: T(\mathrm{HTBKW})=83 \mathrm{~K}, T($ Sternberg $)=10 \mathrm{~K}$. These different temperatures at the $\mathrm{C}^{0}$ abundance peak strongly influences the resulting [CI] surface brightnesses. Overall, the modeldependent surface temperatures still vary significantly. This is due to the additional nonlinearity of the radiative transfer problem, which, under certain circumstances, amplifies even small abundance/temperature differences.

\subsection{Review of participating codes}

It is not our intent to rate the various PDR model codes. Each code was developed with a particular field of application in mind and is capable to fulfill its developers expectations. The restrictions artificially posed by the benchmark standards were additionally limiting the capacity of the participating model codes. Some models encountered for example major numerical difficulties in reaching a stable temperature solution for the benchmark models V4, mainly caused by the requested $\mathrm{H}_{2}$ formation rate of $R=3 \times 10^{-18} T^{1 / 2} \mathrm{~cm}^{3} \mathrm{~s}^{-1}$. This gives reasonable results for low temperatures, but diverges for very high temperatures, resulting in an unreasonably high $\mathrm{H}_{2}$ formation heating. Other codes also show similar numerical problems especially for the model V4. This numerical noise vanishes when we apply more physically reasonable conditions. Nevertheless it was very instructive to study the codes under these extreme conditions.

Every participating code has its own strengths. The Meudon code and Cloudy are certainly the most complex codes in the benchmark, accounting for most physical effects by explicit calculations, starting from the detailed micro-physical processes, making the least use of fitting formulae.

Cloudy was originally developed to simulate extreme environments near accreting black holes (Ferland \& Rees 1988). although it has been applied to HII regions, planetary nebulae, and the ISM. Ferland et al. (1994) describe an early PDR calculation. Its capabilities have been greatly extended over the past several years (van Hoof et al. 2004; Abel et al. 2005; Shaw et al. 2005). Due to the complexity of the code, it was initially not possible to turn off all implemented physical processes as required for the benchmark, but during this study they were able to adopt all benchmark requirements thus removing all major deviations.

The codes HTBKW, Leiden, Sternberg and KOSMA- $\tau$ are based on PDR models that began their development 20 years ago and have been supported and improved since then. One of the main differences between them is the model geometry and illumination. Plane-parallel geometry and uni-directional illumination is assumed in HTBKW, Leiden and Sternberg and spherical geometry with an isotropically impinging FUV field in KOSMA $-\tau$. The chemistry adopted generally in HTBKW is the smallest (46 species) compared with Sternberg (78) and Leiden/KOSMA- $\tau$ (variable). Leiden, Sternberg and KOSMA- $\tau$ explicitly solve the $\mathrm{H}_{2}$ problem (full ro-vib level population) and determine the corresponding shielding by integrating all absorption coefficients while HTBKW uses shielding functions and a single-line approximation for $\mathrm{H}_{2}$. Cloudy is also capable of explicitly calculating a fully $(v, J)$ resolved $\mathrm{H}_{2}$ model, but this capability was switched off in the final model. Instead 

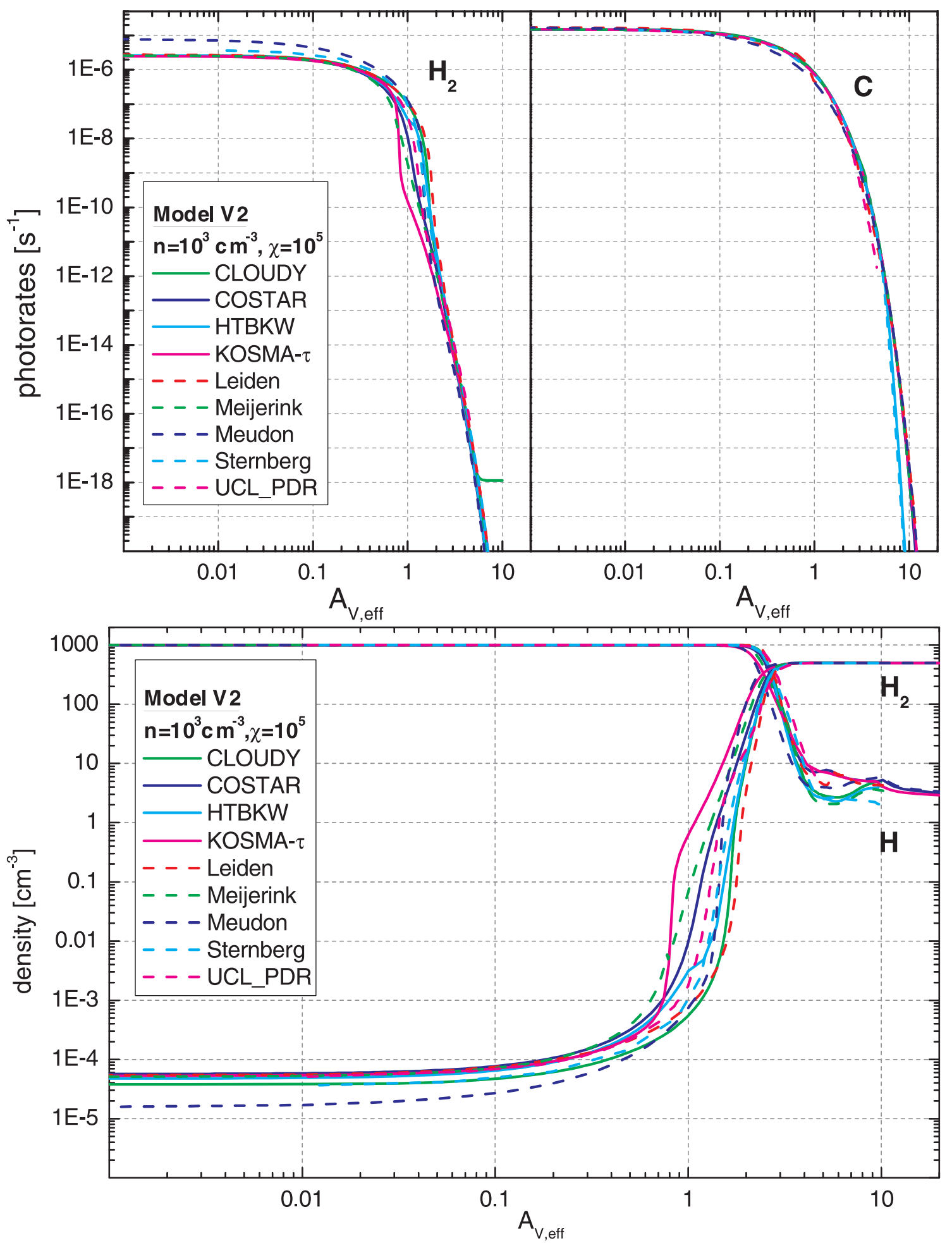

Fig. 13. Model V2 $\left(n=10^{3} \mathrm{~cm}^{-3}, \chi=10^{5}\right)$ : the post-benchmark photo-dissociation rates of $\mathrm{H}_{2}$ (left column), and the photo-ionization rate of $\mathrm{C}$ (right column) (upper plot). The lower plots shows the $\mathrm{H}$ and $\mathrm{H}_{2}$ densities.

they used a 3-level approximation there. Leiden and Meudon are the only codes in the benchmark explicitly calculating the CO shielding, all other codes use shielding factors. HTBKW is additionally accounting for $\mathrm{X}$ ray and PAH heating and computes a large number of observational line intensities, while Leiden focuses on the line emission from the main PDR coolants $\mathrm{C}^{+}$, $\mathrm{C}, \mathrm{O}$, and $\mathrm{CO}$. However it is possible to couple their PDR output with a more sophisticated radiative transfer code such as RATRAN (Hogerheijde \& van der Tak 2000) to calculate emission lines. This is also done by KOSMA- $\tau$, using ONION (Gierens et al. 1992) or SimLine (Ossenkopf et al. 2001). COSTAR was developed in order to model circumstellar disks, featuring any given disk density profile in radial direction and scale height in vertical direction. It uses uni-directional FUV illumination and can treat a surrounding isotropic interstellar FUV field in addition to the uni-directional stellar field. It computes 

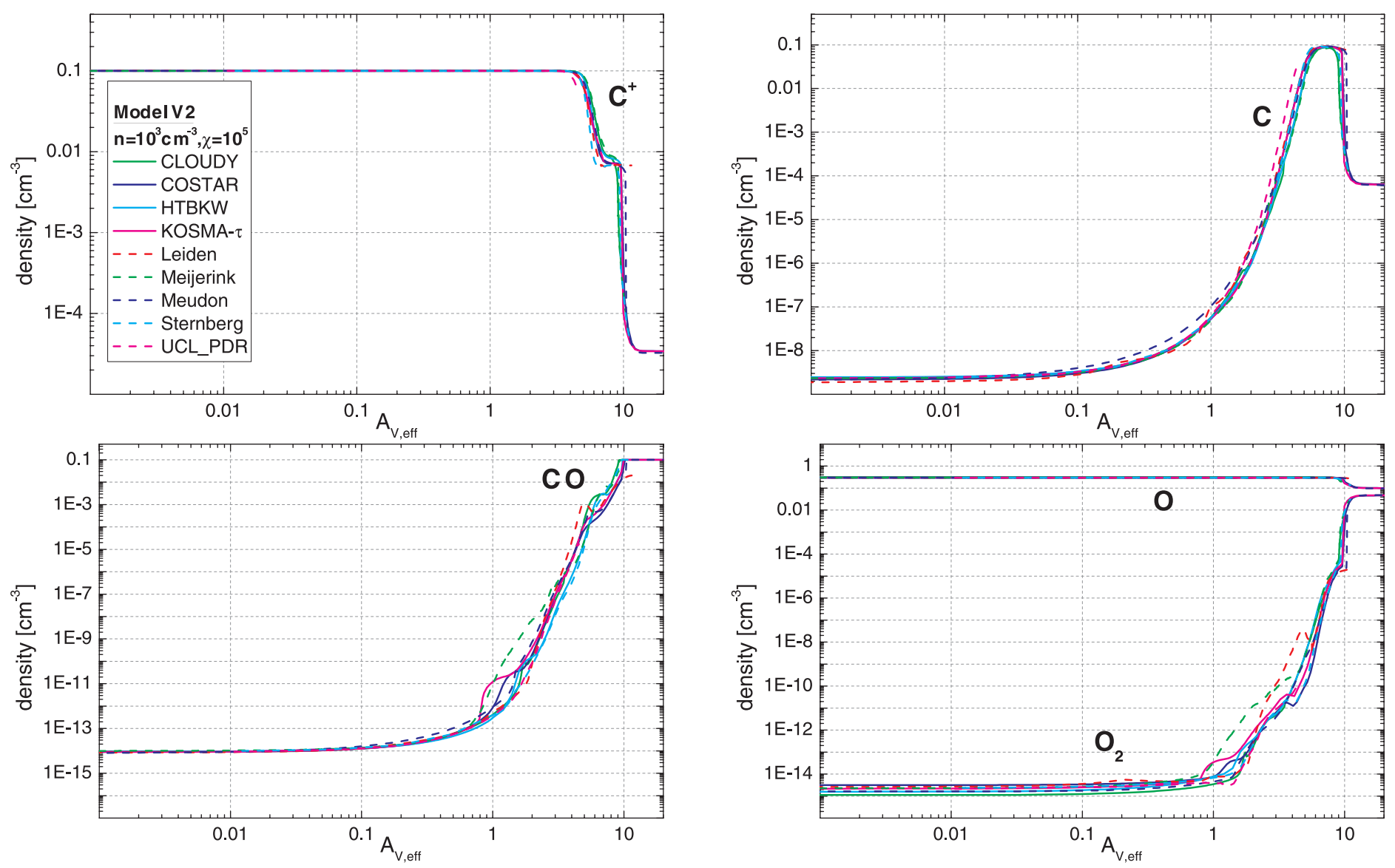

Fig. 14. Model V2 $\left(n=10^{3} \mathrm{~cm}^{-3}, \chi=10^{5}\right)$ : the post-benchmark results for the densities of $\mathrm{C}^{+}$(top left), the densities of $\mathrm{C}$ (top right), and the densities of $\mathrm{CO}$ (bottom left) and $\mathrm{O}$ and $\mathrm{O}_{2}$ (bottom right).

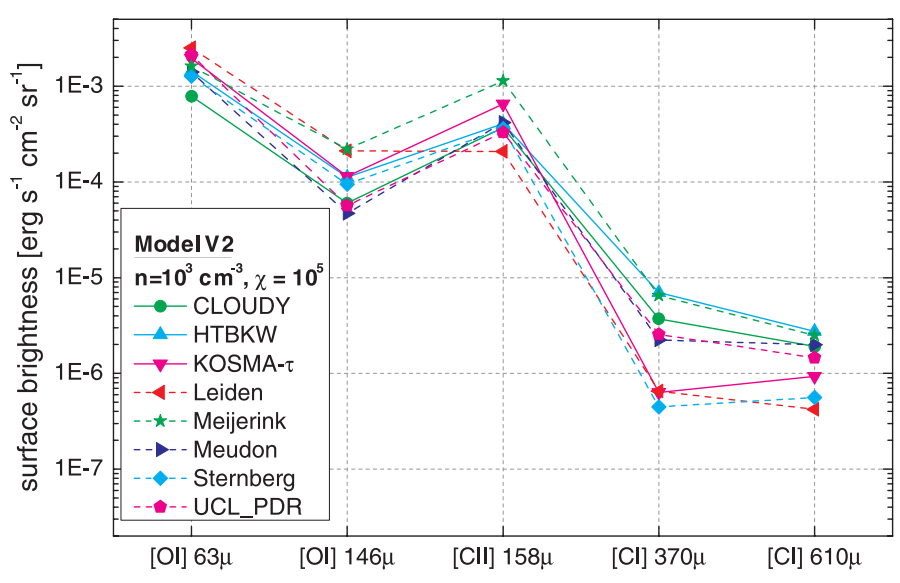

Fig. 15. Model V2 $\left(n=10^{3} \mathrm{~cm}^{-3}, \chi=10^{5}\right)$ : the plot shows the postbenchmark surface brightnesses of the main fine-structure cooling lines: [CII] $158 \mu \mathrm{m},[\mathrm{OI}] 63$, and $146 \mu \mathrm{m}$, and [CI] 610 and $370 \mu \mathrm{m}$.

a relatively small chemical network (48 species) but also accounts for freeze-out onto grains and desorption effects. It relies on shielding functions for $\mathrm{H}_{2}$ and $\mathrm{CO}$ and does not calculate observational line intensities up to now. Nevertheless most of the COSTAR results are in good agreement with the other code results for most of the benchmark models, demonstrating that it correctly accounts for the important PDR physics and chemistry. UCL_PDR is a plane-parallel model focused on time-dependent chemistries with freeze-out and desorption. Its main features are a fully time-dependent treatment - including time-varying density and radiation profiles - and its speed, which makes it suitable for parameter search studies where a large number of models need to be run. It can also be coupled with the SMMOL radiative transfer code (Rawlings \& Yates 2001) for a detailed treatment of the PDR emission properties. Lee96mod was developed from the time-dependent chemical model by Lee, Herbst, and collaborators. It is strongly geared toward complex chemical calculations and only accounts for constant temperatures, neglecting local cooling and heating. Meijerink is a relatively young model with special emphasis on XDRs (X-ray dominated regions) which quickly evolved in the course of this study and we refer to Meijerink \& Spaans (2005) for a detailed review of the current status. In the Appendix we give a tabular overview of all main model characteristics.

\section{Concluding remarks}

We present the latest result in a community wide comparative study among PDR model codes. PDR models are available for almost 30 years now and are established as a common and trusted tool for the interpretation of observational data. The PDR model experts and code-developers have long recognized that the existing codes may deviate significantly in their results, so that observers must not blindly use the output from one of the codes to interpret line observations. The PDR-benchmarking workshop was a first attempt to solve this problem by separating numerical and conceptional differences in the codes, and removing ordinary bugs so that the PDR codes finally turn into a reliable tool for the interpretation of observational data. 


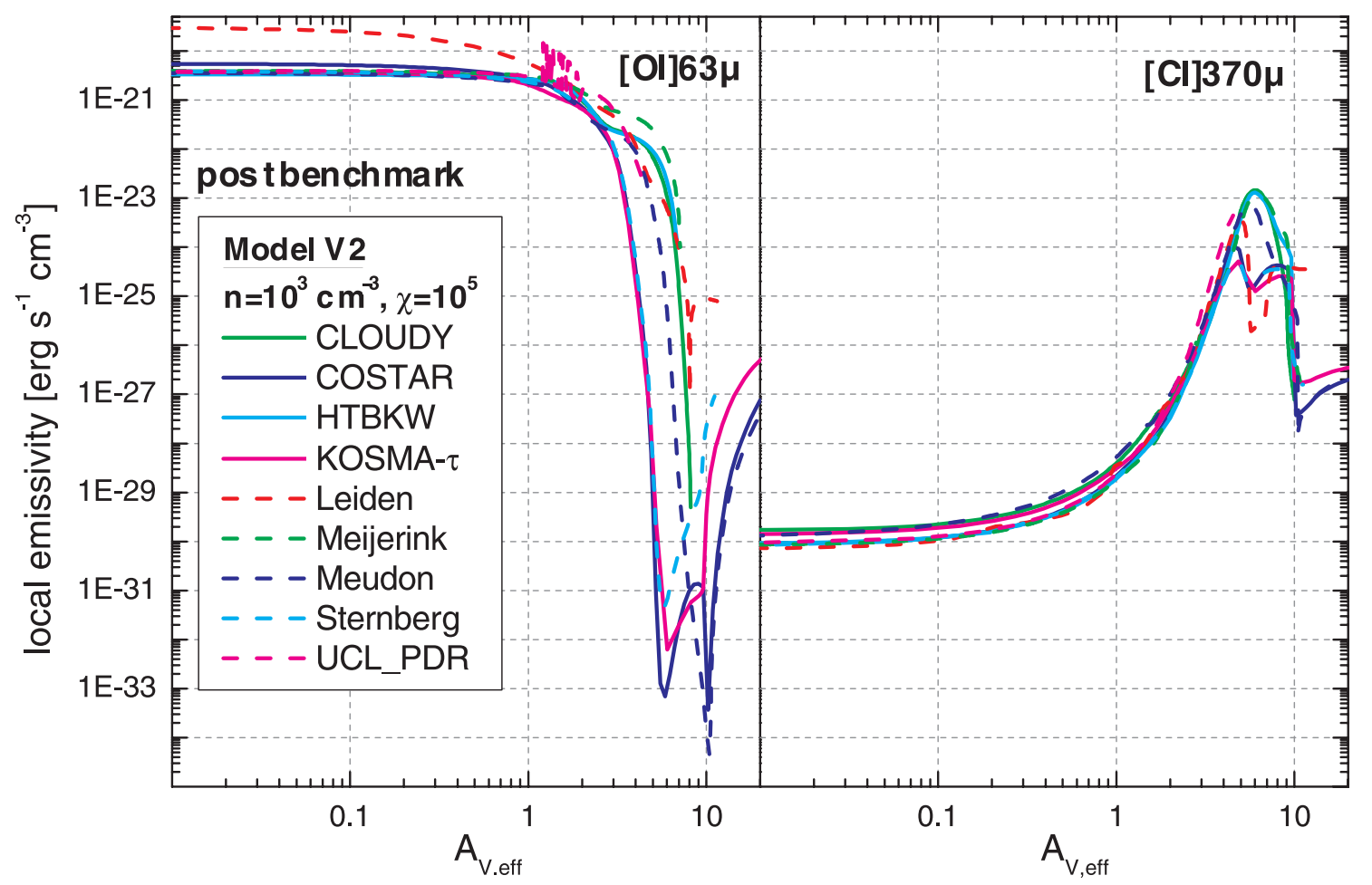

Fig. 16. Model V2 $\left(n=10^{3} \mathrm{~cm}^{-3}, \chi=10^{5}\right)$ : the post-benchmark local emissivities of [OI] $63 \mu \mathrm{m}$ (left column), and [CI] $310 \mu \mathrm{m}$ (right column).

Due to their complex nature it is not always straightforward to compare results from different PDR models with each other. Given the large number of input paramters, it is usually possible to derive more than one set of physical parameters by comparing observations with model predictions, especially when one is chiefly interested in mean densities and temperatures. Our goal was to understand the mutual differences in the different model results and to work toward a better understanding of the key processes involved in PDR modeling. The comparison has revealed the importance of an accurate treatment of various processes, which require further studies.

The workshop and the following benchmarking activities were a success regardless of many open issues. The major results of this study are:

- The collected results from all participating models represent an excellent reference for all present PDR codes and for those to be developed in the future. For the first time such a reference is easily available not only in graphical form but also as raw data. (URL: http: //www .ph1. uni-koeln.de/pdr-comparison)

- We present an overview of the common PDR model codes and summarize their properties and field of application

- As a natural result all participating PDR codes are now better debugged, much better understood, and many differences between the results from different groups are now much clearer resulting in good guidance for further improvements.

- Many critical parameters, model properties and physical processes have been identified or better understood in the course of this study.

- We were able to increase the agreement in model prediction for all benchmark models. Uncertainties still remain, visible e.g. in the deviating temperature profiles of model V2 (Fig. 12) or the large differences for the $\mathrm{H}_{2}$ photo-rates and density profiles in model V4 (cf. online data archive).
- All PDR models are heavily dependent on the chemistry and micro-physics involved in PDRs. Consequently the results from PDR models are only as reliable as the description of the microphysics (rate coefficients, etc.) they are based on.

One of the lessons from this study is that observers should not take the PDR results too literally to constrain, for example, physical parameters like density and radiation field in the region they observe. The current benchmarking shows that all trends are consistent between codes but that there remain differences in absolute values of observables. Moreover it is not possible to simply infer how detailed differences in density or temperature translate into differences in observables. They are the result of a complex, nonlinear interplay between density, temperature, and radiative transfer. We want to emphasize again, that all participating PDR codes are much "smarter" than required during the benchmark. Many sophisticated model features have been switched off in order to provide comparable results. Our intention was technical not physical. The presented results are not meant to model any real astronomical object and should not be applied as such to any such analysis. The current benchmarking results are not meant as our recommended or best values, but simply as a comparison test. During this study we demonstrated, that an increasing level of standardization results in a significant reduction of the model dependent scatter in PDR model predictions. It is encouraging to note the overall agreement in model results. On the other hand it is important to understand that small changes may make a big difference. We were able to identify a number of these key points, e.g. the influence of excited hydrogen, or the importance of secondary photons induced by cosmic rays.

Future work should focus on the energy balance problem, clearly evident from the sometimes significant scatter in the results for the non-isothermal models V1-V4. The heating by photoelectric emission is closely related to the electron density and to the detailed description of grain charges, grain surface 
recombinations and photoelectric yield. The high temperature regime also requires an enlarged set of cooling processes. Another important consideration to be adressed, especially when it comes to comparisons with observations is the model density structure, i.e. clumping or gradients. As a consequence we plan to continue our benchmark effort in the future. This should include a calibration on real observational findings as well.

Acknowledgements. We thank the Lorentz Center, Leiden, for hosting the workshop and for the perfect organization, supplying a very productive environment. The workshop and this work was partly funded by the Deutsche Forschungs Gesellschaft DFG via Grant SFB494 and by a Spinoza grant from the Netherlands Organization for Scientific Research (NWO). We also would like to thank the referee and the editor for making helpful suggestions which helped to improve the manuscript.

\section{References}

Abel, N. P., Ferland, G. J., van Hoof, P. A. M., \& G. Shaw 2005, ApJ, in press [arXiv:astro-ph $\backslash 0506514]$

Abgrall, H., Le Bourlot, J., Pineau Des Forêts, G., et al. 1992, A\&A, 253, 525

Abgrall, H., Roueff, E., Drira, I. 2000, A\&AS, 141, 297

Andriesse, C. D. 1978, A\&A, 66, 196

Arimoto, N., Sofue, Y., \& Tsujimoto, T. 1996, PASJ, 48, 275

Bakes, E. L. O., \& Tielens, A. G. G. M. 1994, ApJ, 427, 822

Bakes, E. L. O., \& Tielens, A. G. G. M. 1998, ApJ, 499, 258

Bell, T. A., Viti, S., Williams, D. A., Crawford, I. A., \& Price, R. J. 2005, MNRAS, 357, 961

Bensch, F., Leuenhagen, U., Stutzki, J., \& Shieder, R. 2003, ApJ, 591, 1913

Bergin, E. A., Melnick, G. J., Stauffer, J. R., et al. 2000, ApJ, 539, L129

Bertoldi, F., \& Draine, B. T 1996, ApJ, 458, 222

Black, J. H., \& Dalgarno, A. 1977, ApJS, 34, 405

Black, J. H., \& van Dishoeck, E. F. 1987, ApJ, 322, 412

Boger, G. I., \& Sternberg, A. 2005, ApJ, 633, 105

Boger, G. I., \& Sternberg, A. 2006, ApJ, in press (see also [arXiv: astro-ph/0601323]

Boissé, P. 1990, A\&A, 228, 483

Bolatto, A. D., Jackson, J. M., \& Ingalls, J. G. 1999, ApJ, 513, 275

Bolatto, A. D., Jackson, J. M., Wilson, C. D., \& Moriarty-Schieven, G. 2000, ApJ, 532, 909

Boselli, A., Lequeux, J., \& Gavazzi, G. 2002a, A\&A, 384, 33

Boselli, A., Gavazzi, G., Lequeux, J., Pierini, D. 2002b, A\&A, 385, 454

Bresolin, F., Garnett, D. R., \& Kennicutt, R. C. 2004, ApJ, 615, 228

Burke, J. R., Hollenbach, D. J. 1983, ApJ, 265, 223

Burton, M. G., Hollenbach, D. J., \& Tielens, A. G. G. M. 1990, ApJ, 365, 620

Cazaux, S., \& Tielens, A. G. G. M. 2004, ApJ, 604, 222

de Boisanger, C. B., Chieze, J.-P., \& Meltz, B. 1992, ApJ, 401, 182

de Jong, T., Boland, W., \& Dalgarno, A. 1980, A\&A, 91, 68

d'Hendecourt, L., \& Léger, A. 1987, A\&A, 180, L9

Dominik, C., Ceccarelli, C., Hollenbach, D., \& Kaufman, M. 2005, ApJ, in press

Draine, B. T. 1978, ApJS, 36, 595

Draine, B. T. 2003, ARA\&A, 41, 241

Draine, B. T., \& Bertoldi, F. 1996, ApJ, 468, 269

Duley et al. 1992, MNRAS, 255, 463

Elmegreen, B. G., \& Falgarone, E. 1996, ApJ, 471, 816

Escalante, V., Sternberg, A., \& Dalgarno, A. 1991, ApJ, 375, 630

Ferland, G. J., \& Rees, M. J. 1988, ApJ, 332, 141

Ferland, G. J., Fabian, A. C., \& Johnstone, R. M. 1994, MNRAS, 266, 399

Ferland, G. J., Korista, K. T., Verner, D. A., et al. 1998, PASP, 110, 761

Flannery, B. P., Roberge, W., \& Rybicki, G. B. 1980, ApJ, 236, 598

Flower, D. R. 1997, MNRAS, 288, 627

Flower, D. R. 1998, MNRAS, 297, 334

Flower, D. R., \& Roueff, E. 1999, MNRAS, 309, 833

Fuente, A., Martin-Pintado, J., Cernicharo, J., \& Bachiller, R. 1993, A\&A, 276, 473

Fuente, A., Martin-Pintado, J., \& Gaume, R. 1995, A\&A, 442, L33

Fuente, A., García-Burillo, S., Gerin, M., et al. 2005, ApJ, 619, L155

Gierens, K. M., Stutzki, J., \& Winnewisser, G. 1992, 259, 271

Glassgold, A. E., \& Langer, W. D. 1975, ApJ, 197, 347

Goldsmith, P. F., Melnick, G. J., Bergin, E. A., et al. 2000, ApJ, 539, L123

Gorti, U., \& Hollenbach, D. 2002, ApJ, 573, 215

Gredel, R., Lepp, S., \& Dalgarno, A., 1987, ApJ, 323, L137

Habing, H. J. 1968, Bull. Astron. Inst. Netherlands, 19, 421

Hegmann, M., \& Kegel, W. H. 1996, MNRAS, 283, 167
Hegmann, M., \& Kegel, W. H. 2003, MNRAS, 342, 453

Heithausen, A., Bensch, F., Stutzki, J., Falgarone, E., \& Panis, J. F. 1998, A\&A, 331, L65

Henyey, L. G., \& Greenstein, J. L. 1941, ApJ, 93, 70

Hill, J. K., \& Hollenbach, D. J. 1978, ApJ, 225, 390

Hogerheijde, M. R., \& van der Tak, F. F. S. 2000, A\&A, 362, 697

Hollenbach, D., \& Salpeter, E. E. 1971, ApJ, 163, 155

Hollenbach, D., \& McKee, C. F. 1979, ApJS, 41, 555

Hollenbach, D., Werner, M. W., \& Salpeter, E. E. 1971, ApJ, 163, 165

Hollenbach, D. J., Takahashi, T., Tielens, A. G. G. M. 1991, ApJ, 377, 192

Hollenbach, D. J., Tielens, A. G. G. M. 1997, ARA\&A, 35, 179

Hollenbach, D. J., Tielens, A. G. G. M. 1999, Rev. Mod. Phys., 71, 173

Howe, J. E., Jaffe, D. T., Genzel, R., \& Stacey, G. J. 1991, ApJ, 373, 158

Hunter, D. A., Kaufman, M., Hollenbach, D. J., et al. 2001, ApJ, 553, 121

Israel, F. P. 1997, A\&A, 328, 471

Israel, F. P., Baas, F., Rudy, R. J., Skillman, E. D., \& Woodward, C. E. 2003, A\&A, 397, 87I

Jansen, D. J., van Dishoeck, E. F., Black, J. H., Spaans, M., \& Sosin, C. 1995, A\&A, 302, 223

Jura, M., 1974, ApJ, 191, 375

Kamp, I., \& Bertoldi, F. 2000, A\&A, 353, 276

Kamp, I., \& van Zadelhoff, G.-J. 2001, A\&A, 373, 641

Kaufman, M. J., Wolfire, M. G., Hollenbach, D. J., \& Luhman, M. L. 1999, ApJ, 527,795

Köster, B., Störzer, H., Stutzki, J., \& Sternberg, A. 1994, A\&A, 284, 545

Kramer, C., Stutzki, J., Rohrig, R., \& Corneliussen, U. 1998, A\&A, 329, 249

Le Bourlot, J., Pineau Des Forêts, G., Roueff, E., \& Flower, D. R. 1993, A\&A, 267,233

Le Bourlot, J. 2006, private communication

Le Petit, F. 2006, A\&A, in press

Le Petit, F., Roueff, E., \& Le Bourlot, J. 2002, A\&A, 390, 369

Le Petit, F., Roeuff, E., \& Herbst, E. 2004, A\&A, 417, 993

Le Teuff, Y. H., Millar, T. J., \& Markwick, A. J. 2000, A\&AS, 146, 157

Lee, H.-H., Herbst, E., Pineau des Forêts, G., Roueff, E., \& Le Bourlot, J. 1996, A\&A, 311, 690

Lepp, S., \& Dalgarno A. 1988, ApJ, 335, 769

Lequeux, J., Le Bourlot, J., Des Forêts, G. P., et al. 1994, A\&A, 292, 371

Li, A., \& Draine, B. T. 2002, ApJ, 576, 762

Lintott, C. J., \& Rawlings, J. M. C. 2006, A\&A, 448, 425

Liseau, R., White, G. J., Larsson, B., et al. 1999, A\&A, 344, 342

Madden, S. C., Poglitsch, A., Geis, N., Stacey, G. J., \& Townes, C. H. 1997, ApJ, 483, 200

Madden, S. C. 2000, NewAR, 44, 249

Markwick-Kemper, A. J. 2005, IAU Symp. 231, ed. A. J. Markwick-Kemper

Meijerink, R., \& Spaans, M. 2005, A\&A, 436, 397

McKee, C. F., 1989, ApJ, 345, 782

Meixner, M., \& Tielens, A. G. G. M. 1993, ApJ, 405, 216

Millar, T. J., Farquhar, P. R. A., \& Willacy, K. 1997, A\&AS, 121, 139

Mochizuki, K., Onaka, T., \& Nakagawa, T. 1998, ASP Conf. Ser., 132, 386

Nejad, L. A. M., Wagenblast, R. 1999, A\&A, 350, 204

Ossenkopf, V., Trojan, C., \& Stutzki, J. 2001, A\&A, 378, 608

Pagani, L., Olofsson, A. O. H., Bergmann, P., et al. 2003, A\&A, 402, L69

Pak, S., Jaffe, D. T., van Dishoeck, E. F., Johansson, L. E. B., \& Booth, R. S. 1998, ApJ, 498, 735

Papadopoulos, P. P., Thi, W.-F., Viti, S. 2002, ApJ, 579, 270

Rawlings, J. M. C., Yates, J. A. 2001, MNRAS, 326, 1423

Röllig, M., Hegmann, M., Kegel, W. H. 2002, A\&A, 392, 1081

Röllig, M., Ossenkopf, V., Jeyakumar, S., Stutzki, J., Sternberg, A. 2006, A\&A, 451,917

Roberge, W. G., Jones, D., Lepp, S., \& Dalgarno, A. 1991, ApJS, 77, 287

Roberts, H., \& Herbst, E., A\&A, 395, 233

Rubio, M., Boulanger, F., Rantakyro, F., \& Contursi, A. 2004, A\&A, 425, L1

Savage, C., \& Ziurys, L. M. 2004, ApJ, 616, 966

Shaw, G., Ferland, G. J., Abel, N. P., Stancil, P., \& van Hoof P. A. M. 2005, ApJ, 624,794

Shalabiea, O. M., \& Greenberg, J. M. 1995, A\&A, 296, 779

Simon, R., Jackson, J. M., Clemens, D. P., Bania, T. M., \& Heyer, M. H. 2001, ApJ, 551, 747

Spaans, M. 1996, A\&A, 307, 271

Spaans, M., \& van Dishoeck, E. F. 1997, A\&A, 323, 953

Spaans, M., \& van Dishoeck, E. F. 2001, ApJ, 548, L217

Stacey, G. J., Geis, N., Genzel, R., et al. 1991, ApJ, 373, 423

Steiman-Cameron, T. Y., Haas, M. R., Tielens, A. G. G. M., \& Burton, M. G. 1997, ApJ, 478, 261

Sternberg, A. 1988, ApJ, 332, 400

Sternberg, A. 1989, ApJ, 347, 863

Sternberg, A. 1990, ApJ, 361, 121

Sternberg, A., \& Dalgarno, A. 1989, ApJ, 338, 197 
Sternberg, A., \& Dalgarno, A. 1995, ApJS, 99, 565

Sternberg, A., \& Neufeld, D. A. 1999, ApJ, 516, 371

Störzer, H., Stutzki, J., \& Sternberg, A. 1996, A\&A, 310, 592

Störzer, H., \& Hollenbach, D. 1998, ApJ, 495, 853

Störzer, H., Zielinsky, M., Stutzki, J., \& Sternberg, A. 2000, A\&A, 358, 682

Stutzki, J. 1984, Ph. D. Thesis, Universität zu Köln

Stutzki, J., Stacey, G. J., Genzel, R., et al. 1988, ApJ, 332, 379

Taylor, S. D., Hartquist, T. W., \& Williams, D. A. 1993, MNRAS, 264, 929

Terzieva, R., \& Herbst, E. 1998, ApJ, 501, 207

Teyssier, D., Fossé, D., Gerin, M., et al. 2004, A\&A, 417, 135

Thuan, T. X., Sauvage, M., \& Madden, S. 1999, ApJ, 516, 783

Tielens, A. G. G. M., Hollenbach, D. 1985, ApJ, 291, 722

Trumpler, R. J. 1930, PASP, 42, 267

van Dishoeck, E. F. 1988, in Rate Coefficients in Astrochemistry, ed. T. J. Millar,

\& D. A. Williams (Dordrecht: Kluwer Academic Publishers), 49

van Dishoeck, E. F., Black, \& John, H. 1988, ApJ, 334, 771

van Hoof, P. A. M., Weingartner, J. C., Martin, P. G., Volk, K., \& Ferland, G. J. 2004, MNRAS, 350, 1330

van Zadelhoff, G.-J., Dullemond, C. P., van der Tak, F. F. S., et al. 2002, A\&A, 395,373
Verstrate, L., Léger, A., d'Hendecourt, L., Dutuit, O., \& Defourneau, D. 1990, A\&A, 237, 436

Viti, S., Roueff, E., Hartquist, T. W., Pineau des Forêts, G., \& Williams, D. A. 2001, A\&A, 370, 557

Weingartner, J. C., \& Draine, B. T. 2001, ApJ, 563, 842

Wagenblast, R., \& Hartquist, T. W. 1988, MNRAS, 230, 363

Wakelam, V. 2004, A\&A, 413, 609

Wakelam, V., Selsis, F., Herbst, E., \& Caselli, P. 2005a, A\&A, submitted

Wakelam, V., Herbst, E., \& Selsis, F. 2005b, IAU Symp. 231, ed. A. J. MarkwickKemper

Walmsley, C. M., Pineau des Forêts, G., Flower, D. R. 1999, A\&A, 342, 542

Warin, S., Benayoun, J. J., \& Viala, Y. P. 1996, A\&A, 308, 535

Wilson, C. D. 1995, ApJ, 448, L97

Wilson, C. D., Olofsson, A. O. H., Pagani, L., et al. 2005, A\&A, 433, L5

Wolfire, M. G., Hollenbach, D., McKee, C. F., Tielens, A. G. G. M., \& Bakes, E. L. O. 1995, ApJ, 443, 152

Wolfire, M. G., McKee, C. F., Hollenbach, D., \& Tielens, A. G. G. M. 2003, ApJ, 587,278

Zaritsky, D., Kennicutt, R. C., Jr., \& Huchra, J. P. 1994, ApJ, 420, 87

Zielinsky, M., Stutzki J., \& Störzer, H. 2000, A\&A, 358 
M. Röllig et al.: A photon dominated region code comparison study, Online Material p 1

\section{Online Material}


M. Röllig et al.: A photon dominated region code comparison study, Online Material p 2

\section{Appendix A: Characteristics of participating PDR}

codes

In Table A.1 we summarize the most important characteristics of the participating PDR codes. This table summarizes the full capabilities of the PDR codes and is not limited to the benchmark standards. It has been extracted from detailed characteristics sheets, available online for all codes: http://www . ph1. uni-koeln.de/pdr-comparison. 
M. Röllig et al.: A photon dominated region code comparison study, Online Material p 3

Table A.1. Full capabilities of the PDR model codes participating in the Leiden comparison study.

\begin{tabular}{|c|c|c|c|c|c|c|c|c|c|c|c|}
\hline & $\frac{\overrightarrow{0}}{0}$ & $\underset{0}{\mathscr{U}}$ & 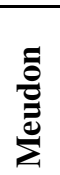 & 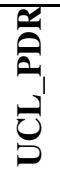 & $\frac{3}{3}$ & 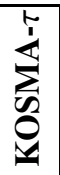 & $\frac{\pi}{7}$ & 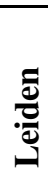 & 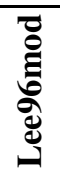 & 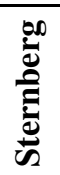 & 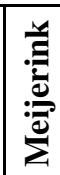 \\
\hline
\end{tabular}

\section{GEOMETRY}

\begin{tabular}{l|c|c|c|c|c|c|c|c|c|c|c}
\hline spherical & $\mathrm{X}$ & & & & & $\mathrm{X}$ & & & & & \\
\hline plane-parallel, finite & $\mathrm{X}$ & & $\mathrm{X}$ & & $\mathrm{X}$ & & & $\mathrm{X}$ & & & \\
\hline plane-parallel, semi-infinite & $\mathrm{X}$ & & $\mathrm{X}$ & $\mathrm{X}$ & $\mathrm{X}$ & & $\mathrm{X}$ & $\mathrm{X}$ & $\mathrm{X}$ & $\mathrm{X}$ & $\mathrm{X}$ \\
\hline circumstellar disc & $\mathrm{X}$ & $\mathrm{X}$ & & & & & & $\mathrm{X}$ & & & \\
\hline ensemble of clouds & & & & & & $\mathrm{X}$ & & & & & \\
\hline
\end{tabular}

DENSITY

\begin{tabular}{|c|c|c|c|c|c|c|c|c|c|c|c|}
\hline homogeneous & $\mathrm{X}$ & $\mathrm{X}$ & $\mathrm{X}$ & $\mathrm{X}$ & $\mathrm{X}$ & $\mathrm{X}$ & $\mathrm{X}$ & $\mathrm{X}$ & $\mathrm{X}$ & $\mathrm{X}$ & $\mathrm{X}$ \\
\hline density-law & $\mathrm{X}$ & $\mathrm{x}$ & $\mathrm{X}$ & $\mathrm{X}$ & & $\mathrm{X}$ & $\mathrm{x}$ & $\mathrm{x}$ & $\mathrm{x}$ & $\mathrm{x}$ & \\
\hline time dependent & $\mathrm{X}$ & & & $\mathrm{X}$ & & & & & & & \\
\hline velocity field & $\mathrm{X}$ & & & & & $\mathrm{X}$ & & & & & \\
\hline$v=$ const & $\mathrm{x}$ & & & & & $\mathrm{X}$ & & & & & \\
\hline$v=v(r, \ldots)$ & $\mathrm{X}$ & & & & & & & & & & \\
\hline
\end{tabular}

RADIATION

\begin{tabular}{|c|c|c|c|c|c|c|c|c|c|c|c|}
\hline isotropic radiation field & & & $\mathrm{X}$ & & & $\mathrm{X}$ & & & & & \\
\hline uni-directional radiation field & $\mathrm{x}$ & $\mathrm{x}$ & $\mathrm{X}$ & $\mathrm{X}$ & $\mathrm{x}$ & & $\mathrm{x}$ & $\mathrm{x}$ & $\mathrm{X}$ & $\mathrm{x}$ & $\mathrm{x}$ \\
\hline combination of isotropic+illuminating star & & & $\mathrm{X}$ & & & & & & & & \\
\hline & & & & & & & & & & & \\
\hline Habing field & $\mathrm{x}$ & & & $\mathrm{X}$ & $\mathrm{x}$ & & & $\mathrm{x}$ & & & $\mathrm{X}$ \\
\hline Draine field & $\mathrm{x}$ & $\mathrm{X}$ & $\mathrm{X}$ & & $\mathrm{X}$ & $\mathrm{X}$ & & $\mathrm{X}$ & & $\mathrm{X}$ & \\
\hline optional star & $\mathrm{X}$ & & $\mathrm{X}$ & & & & & $\mathrm{X}$ & & & \\
\hline detailed SED & $\mathrm{x}$ & & $\mathrm{x}$ & & & & & & & & \\
\hline other & $\mathrm{X}$ & & & & & & $\mathrm{X}$ & $\mathrm{X}$ & $\mathrm{x}$ & & \\
\hline external radiation source & $\mathrm{x}$ & $\mathrm{x}$ & $\mathrm{X}$ & $\mathrm{x}$ & $\mathrm{x}$ & $\mathrm{x}$ & $\mathrm{x}$ & $\mathrm{x}$ & $\mathrm{x}$ & $\mathrm{x}$ & $\mathrm{x}$ \\
\hline internal radiation source & & & & & & & & & & & \\
\hline
\end{tabular}

\section{CHEMISTRY}

\begin{tabular}{|c|c|c|c|c|c|c|c|c|c|c|c|}
\hline stationary chemistry & $\mathrm{x}$ & $\mathrm{x}$ & $\mathrm{x}$ & & $\mathrm{x}$ & $\mathrm{x}$ & & $\mathrm{x}$ & & $\mathrm{x}$ & $\mathrm{x}$ \\
\hline time-dependent chemistry & $\mathrm{X}$ & & & $\mathrm{x}$ & & & $\mathrm{x}$ & & $\mathrm{x}$ & & \\
\hline advection flow & $\mathrm{X}$ & & & & & & & & & & \\
\hline & & & & & & & & & & & \\
\hline UMIST95 & $\mathrm{X}$ & & $\mathrm{X}$ & $\mathrm{x}$ & & $\mathrm{X}$ & & $\mathrm{X}$ & & $\mathrm{X}$ & $\mathrm{X}$ \\
\hline UMIST99 & $\mathrm{X}$ & & & $\mathrm{X}$ & $\mathrm{X}$ & $\mathrm{x}$ & & & & $\mathrm{X}$ & $\mathrm{X}$ \\
\hline NSM & & & $\mathrm{X}$ & & & & $\mathrm{x}$ & & $\mathrm{X}$ & & \\
\hline other database & $\mathrm{X}$ & $\mathrm{X}$ & $\mathrm{X}$ & & & $\mathrm{x}$ & & $\mathrm{x}$ & & $\mathrm{X}$ & \\
\hline fixed number of species & $\mathrm{x}$ & $\mathrm{x}$ & & $\mathrm{X}$ & $\mathrm{x}$ & & $\mathrm{X}$ & & $\bar{X}$ & $\mathrm{X}$ & \\
\hline variable number of species & & & $\mathrm{x}$ & & & $\mathrm{x}$ & & $\mathrm{x}$ & & & $\mathrm{x}$ \\
\hline number of species & 96 & 48 & & 128 & 46 & & 577 & & 419 & 78 & \\
\hline PAH's included & $\mathrm{X}$ & & $\mathrm{X}$ & & $\mathrm{X}$ & $\mathrm{X}$ & & $\mathrm{X}$ & & & $\mathrm{X}$ \\
\hline & & & & & & & & & & & \\
\hline freeze-out on grains included & $\mathrm{x}$ & $\mathrm{x}$ & $\mathrm{X}$ & $\mathrm{x}$ & & & $\mathrm{x}$ & & & & \\
\hline$\overline{\mathrm{H}_{2} \text { formation on grains }}$ & $\mathrm{X}$ & $\mathrm{X}$ & $\mathrm{X}$ & $\mathrm{X}$ & $\mathrm{X}$ & $\mathrm{X}$ & $\mathrm{X}$ & $\bar{x}$ & $\bar{X}$ & $\mathrm{X}$ & $\mathrm{x}$ \\
\hline formation of other molecules on grains & & & $\mathrm{X}$ & $\mathrm{X}$ & & & $\mathrm{X}$ & & & & \\
\hline & & & & & & & & & & & \\
\hline desorption mechanisms included & & $\mathrm{x}$ & $\mathrm{x}$ & & & & $\mathrm{x}$ & & & & \\
\hline thermal desorption & $\mathrm{X}$ & $\mathrm{X}$ & & & & & $\mathrm{x}$ & & & & \\
\hline photoevaporation & & & $\mathrm{x}$ & & & & & & & & \\
\hline CR spot heating & $\mathrm{X}$ & & $\mathrm{X}$ & & & & $\mathrm{X}$ & & & & \\
\hline
\end{tabular}


Table A.1. continued.

\begin{tabular}{|c|c|c|c|c|c|c|c|c|c|c|c|}
\hline & $\begin{array}{l}\frac{\partial}{0} \\
\frac{0}{0}\end{array}$ & 䒘 & $\begin{array}{l}\text { 를 } \\
\text { 를 }\end{array}$ & 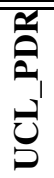 & $\frac{1}{3}$ & 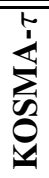 & 苞 & 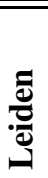 & 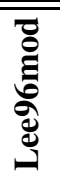 & 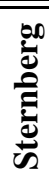 & 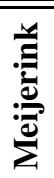 \\
\hline grain-grain collisions & & & $\mathrm{X}$ & & & & & & & & \\
\hline metallicity included & $\mathrm{X}$ & $\mathrm{x}$ & $\mathrm{x}$ & $\mathrm{X}$ & $\mathrm{X}$ & $\mathrm{X}$ & & $\mathrm{X}$ & & $\mathrm{X}$ & $\mathrm{X}$ \\
\hline
\end{tabular}

ISOTOPOMERS

\begin{tabular}{c|c|c|c|c|c|c|c|c|c|c|c|c}
\hline${ }^{13} \mathrm{C}$ & $\mathrm{x}$ & & $\mathrm{x}$ & & & $\mathrm{x}$ & & $\mathrm{x}$ & & & $\mathrm{x}$ \\
\hline${ }^{17} \mathrm{O}$ & & & & & & & & & & & \\
\hline${ }^{18} \mathrm{O}$ & & & $\mathrm{x}$ & & & $\mathrm{x}$ & & $\mathrm{x}$ & & & \\
\hline $\mathrm{D}$ & $\mathrm{x}$ & & $\mathrm{x}$ & & & & $\mathrm{x}$ & $\mathrm{x}$ & & & \\
\hline & & & & & & & & & & & \\
\hline
\end{tabular}

THERMAL BALANCE

\begin{tabular}{l|c|c|c|c|c|c|c|c|c|c|c}
\hline fixed temperature & $\mathrm{X}$ & $\mathrm{x}$ & $\mathrm{x}$ & $\mathrm{X}$ & & $\mathrm{x}$ & & $\mathrm{x}$ & $\mathrm{x}$ & $\mathrm{x}$ & $\mathrm{x}$ \\
\hline temperature determined from energy balance & $\mathrm{X}$ & $\mathrm{x}$ & $\mathrm{x}$ & $\mathrm{X}$ & $\mathrm{x}$ & $\mathrm{x}$ & $\mathrm{X}$ & $\mathrm{x}$ & & $\mathrm{x}$ & $\mathrm{x}$ \\
\hline
\end{tabular}

\section{COOLING}

\begin{tabular}{|c|c|c|c|c|c|c|c|c|c|c|}
\hline gas-grain cooling & $\mathrm{x}$ & $\mathrm{x}$ & $\mathrm{x}$ & $\mathrm{X}$ & $\mathrm{X}$ & $\mathrm{X}$ & $\mathrm{X}$ & $\mathrm{X}$ & $\mathrm{X}$ & $\mathrm{x}$ \\
\hline radiative recombination & $\mathrm{x}$ & & $\mathrm{X}$ & & & $\mathrm{X}$ & & & $\mathrm{x}$ & \\
\hline \multirow[t]{2}{*}{ chemical balance } & & & $\mathrm{X}$ & & & & & & & \\
\hline & & & & & & & & & & \\
\hline [OI] lines & $\mathrm{x}$ & $\mathrm{X}$ & $\mathrm{X}$ & $\mathrm{X}$ & $\mathrm{X}$ & $\mathrm{X}$ & $\mathrm{X}$ & $\mathrm{X}$ & $\mathrm{X}$ & $\mathrm{x}$ \\
\hline${ }^{12} \mathrm{CO}$ rotational lines & $\mathrm{x}$ & $\mathrm{X}$ & $\mathrm{X}$ & $\mathrm{X}$ & $\mathrm{X}$ & $\mathrm{x}$ & $\mathrm{X}$ & $\mathrm{X}$ & & $\mathrm{x}$ \\
\hline${ }^{13} \mathrm{CO}$ rotational lines & $\mathrm{x}$ & & $\mathrm{X}$ & & & $\mathrm{x}$ & $\mathrm{X}$ & & & $\bar{x}$ \\
\hline [CII] line & $\mathrm{X}$ & $\mathrm{x}$ & $\mathrm{X}$ & $\mathrm{x}$ & $\mathrm{X}$ & $\mathrm{X}$ & $\mathrm{x}$ & $\mathrm{X}$ & $\mathrm{X}$ & $\mathrm{x}$ \\
\hline [CI] lines & $\mathrm{X}$ & $\mathrm{X}$ & $\mathrm{X}$ & $\mathrm{X}$ & $\mathrm{X}$ & $\mathrm{X}$ & $\mathrm{X}$ & $\mathrm{X}$ & $\mathrm{X}$ & $\bar{x}$ \\
\hline [SiII] lines & $\mathrm{X}$ & & $\mathrm{X}$ & & $\mathrm{X}$ & $\mathrm{X}$ & & & $\mathrm{X}$ & $\mathrm{x}$ \\
\hline $\mathrm{OH}$ rotational lines & & & $\mathrm{X}$ & & $\mathrm{X}$ & $\mathrm{x}$ & & & $\mathrm{X}$ & $\mathrm{x}$ \\
\hline $\mathrm{H}_{2} \mathrm{O}$ rotational lines & & & $\mathrm{X}$ & & $\mathrm{X}$ & $\mathrm{x}$ & & & $\mathrm{X}$ & $\bar{x}$ \\
\hline $\mathrm{H}_{2}$ rotational lines & $\mathrm{x}$ & & $\mathrm{X}$ & $\mathrm{X}$ & & & & & $\mathrm{x}$ & $\mathrm{x}$ \\
\hline HD rotational lines & $\mathrm{X}$ & & $\mathrm{X}$ & & & & & & & \\
\hline [OI] 6300^ metastable lines & $\mathrm{x}$ & $\mathrm{x}$ & $\mathrm{x}$ & $\mathrm{x}$ & & & & $\mathrm{x}$ & $\mathrm{x}$ & $\mathrm{x}$ \\
\hline $\mathrm{CH}$ rotational lines & & $\mathrm{X}$ & & & & & & & $\mathrm{X}$ & \\
\hline Ly $\alpha$ metastable lines & $\mathrm{X}$ & $\mathrm{x}$ & & $\mathrm{X}$ & & & & $\mathrm{X}$ & & $\mathrm{X}$ \\
\hline $\mathrm{Fe}(24 \mu, 34 \mu),[\mathrm{FeII}](26 \mu, 35.4 \mu)$ & $\mathrm{X}$ & & & & $\mathrm{X}$ & & & & $\mathrm{X}$ & $\mathrm{x}$ \\
\hline $\mathrm{H}_{2}$ (rot-vib) & $\mathrm{X}$ & & $\mathrm{x}$ & & $\mathrm{X}$ & & & & $\mathrm{x}$ & $\mathrm{x}$ \\
\hline
\end{tabular}

\section{HEATING}

\begin{tabular}{|c|c|c|c|c|c|c|c|c|c|c|}
\hline $\mathrm{H}_{2}$ vibrational deexcitation & $\mathrm{X}$ & $\mathrm{X}$ & $\mathrm{X}$ & $\mathrm{X}$ & $\mathrm{X}$ & $\mathrm{X}$ & $\mathrm{X}$ & $\mathrm{X}$ & $\mathrm{X}$ & $\mathrm{X}$ \\
\hline single line approx. & $\mathrm{X}$ & $\mathrm{X}$ & & $\mathrm{X}$ & $\mathrm{X}$ & & $\mathrm{X}$ & $\mathrm{X}$ & & $\mathrm{X}$ \\
\hline only n-levels, but no J & & & & & & $\mathrm{X}$ & & & $\mathrm{X}$ & \\
\hline full rot-vib treatment & $\mathrm{X}$ & & $\mathrm{X}$ & & & & & & $\mathrm{X}$ & \\
\hline $\mathrm{H}_{2}$ dissociation & $\mathrm{X}$ & $\mathrm{X}$ & $\mathrm{X}$ & $\mathrm{X}$ & $\mathrm{x}$ & $\mathrm{x}$ & $\mathrm{X}$ & $\mathrm{X}$ & $\mathrm{X}$ & $\mathrm{X}$ \\
\hline $\mathrm{H}_{2}$ formation & $\mathrm{x}$ & $\mathrm{X}$ & $\mathrm{X}$ & $\mathrm{X}$ & $\mathrm{X}$ & $\mathrm{X}$ & & $\mathrm{x}$ & $\mathrm{X}$ & $\mathrm{X}$ \\
\hline CR heating & $\mathrm{X}$ & $\mathrm{X}$ & $\mathrm{X}$ & $\mathrm{X}$ & $\mathrm{X}$ & $\mathrm{X}$ & $\mathrm{X}$ & $\mathrm{X}$ & $\mathrm{X}$ & $\mathrm{X}$ \\
\hline PE heating & $\mathrm{X}$ & $\mathrm{x}$ & $\mathrm{X}$ & $\mathrm{X}$ & $\mathrm{X}$ & $\mathrm{X}$ & $\mathrm{x}$ & $\mathrm{x}$ & $\mathrm{X}$ & $\mathrm{X}$ \\
\hline XR heating & $\mathrm{X}$ & & & $\mathrm{X}$ & $\mathrm{X}$ & & & & & $\mathrm{X}$ \\
\hline PAH heating & $\mathrm{X}$ & & $\mathrm{X}$ & $\mathrm{X}$ & $\mathrm{X}$ & $\mathrm{X}$ & & $\mathrm{x}$ & & $\mathrm{X}$ \\
\hline photoionization & $\mathrm{X}$ & $\mathrm{X}$ & $\mathrm{X}$ & $\mathrm{X}$ & & & & $\mathrm{X}$ & & $\mathrm{X}$ \\
\hline carbon ionization heating & $\mathrm{x}$ & $\mathrm{X}$ & $\mathrm{X}$ & $\mathrm{X}$ & & & & $\mathrm{X}$ & $\mathrm{X}$ & $\mathrm{X}$ \\
\hline other species $(\mathrm{Si}$, etc.) & $\mathrm{x}$ & & $\mathrm{X}$ & & & & & & & \\
\hline gas-grain collisions & $\mathrm{x}$ & $\mathrm{x}$ & $\mathrm{X}$ & $\mathrm{X}$ & & & & $\mathrm{X}$ & $\mathrm{X}$ & $\mathrm{X}$ \\
\hline turbulence heating & $\mathrm{X}$ & & $\mathrm{X}$ & $\mathrm{X}$ & & & & & & \\
\hline chemical balance & & & $\mathrm{X}$ & $\mathrm{X}$ & & & & $\mathrm{X}$ & & \\
\hline
\end{tabular}


Table A.1. continued.

\begin{tabular}{|c|c|c|c|c|c|c|c|c|c|c|c|}
\hline & 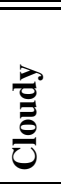 & $\begin{array}{l}\frac{\pi}{1} \\
0 \\
0 \\
0\end{array}$ & 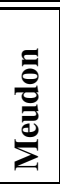 & 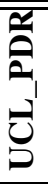 & 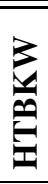 & 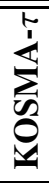 & 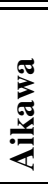 & : & 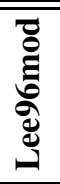 & 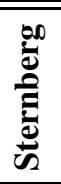 & 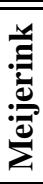 \\
\hline solved self-consistently & $\mathrm{x}$ & $\mathrm{x}$ & $\mathrm{x}$ & $\mathrm{x}$ & $\mathrm{x}$ & $\mathrm{x}$ & & $\mathrm{x}$ & & $\mathrm{X}$ & $\mathrm{x}$ \\
\hline simple exponential attenuation & $\mathrm{X}$ & $\mathrm{x}$ & $\mathrm{x}$ & $\mathrm{x}$ & $\mathrm{X}$ & $\mathrm{X}$ & $\mathrm{x}$ & $\mathrm{X}$ & $\mathrm{x}$ & $\mathrm{x}$ & $\mathrm{X}$ \\
\hline bi-exponential attenuation & & $\mathrm{x}$ & & & & & & & & $\mathrm{x}$ & \\
\hline full RT in lines & & & $\mathrm{x}$ & & & & & $\mathrm{x}$ & & & \\
\hline \multicolumn{12}{|c|}{ DUST } \\
\hline treatment of rad. transfer & $\mathrm{x}$ & & $\mathrm{X}$ & & $\mathrm{x}$ & $\mathrm{X}$ & & $\mathrm{x}$ & & $\mathrm{X}$ & $\mathrm{x}$ \\
\hline grain size distribution & $\mathrm{x}$ & & $\mathrm{X}$ & $\mathrm{X}$ & & $\mathrm{X}$ & & & & & \\
\hline extinction/scattering law & $\mathrm{x}$ & $\mathrm{X}$ & $\mathrm{X}$ & $\mathrm{X}$ & $\mathrm{x}$ & $\mathrm{x}$ & $\mathrm{x}$ & $\mathrm{x}$ & $\mathrm{x}$ & $\mathrm{X}$ & \\
\hline albedo & $\mathrm{x}$ & & $\mathrm{X}$ & $\mathrm{X}$ & & & & $\mathrm{X}$ & & & $\mathrm{X}$ \\
\hline scattering law & $\mathrm{X}$ & & $\mathrm{X}$ & & & & & $\mathrm{X}$ & & & \\
\hline
\end{tabular}

\section{$\mathrm{H}_{2}$ SHIELDING}

\begin{tabular}{l|c|c|c|c|c|c|c|c|c|c|c}
\hline shielding factors & $\mathrm{X}$ & $\mathrm{X}$ & & & $\mathrm{X}$ & & $\mathrm{X}$ & & $\mathrm{X}$ & $\mathrm{X}$ & $\mathrm{X}$ \\
\hline single line & $\mathrm{X}$ & & & $\mathrm{X}$ & & & & & & & $\mathrm{X}$ \\
\hline detailed solution & $\mathrm{X}$ & & $\mathrm{X}$ & & & $\mathrm{X}$ & & $\mathrm{X}$ & & & \\
\hline
\end{tabular}

\section{CO SHIELDING}

\begin{tabular}{l|c|c|c|c|c|c|c|c|c|c|c}
\hline shielding factors & $\mathrm{X}$ & $\mathrm{X}$ & & $\mathrm{X}$ & $\mathrm{X}$ & $\mathrm{X}$ & $\mathrm{X}$ & $\mathrm{X}$ & $\mathrm{X}$ & $\mathrm{X}$ & $\mathrm{X}$ \\
\hline single line & $\mathrm{X}$ & & & & & & & $\mathrm{X}$ & & & $\mathrm{X}$ \\
\hline detailed solution & & & $\mathrm{X}$ & & & & & $\mathrm{X}$ & & & \\
\hline isotope selective photodissociation & & & $\mathrm{X}$ & & & $\mathrm{X}$ & & $\mathrm{X}$ & & & $\mathrm{X}$ \\
\hline
\end{tabular}

\section{UV PROFILE FUNCTION}

\begin{tabular}{l|l|l|l|l|l|l|l|l|l|l|l}
\hline Gaussian & & & & $\mathrm{X}$ & $\mathrm{X}$ & & & & & & \\
\hline Voigt & $\mathrm{X}$ & & $\mathrm{X}$ & & & & & $\mathrm{X}$ & & $\mathrm{X}$ & $\mathrm{X}$ \\
\hline Box & & & & & & & & & & & \\
\hline other & & & & & & & & & & & \\
\hline
\end{tabular}

\section{RADIATIVE TRANSFER IN COOLING LINES}

\begin{tabular}{l|c|c|c|c|c|c|c|c|c|c|c}
\hline escape probability & $\mathrm{X}$ & $\mathrm{X}$ & $\mathrm{X}$ & $\mathrm{X}$ & $\mathrm{X}$ & $\mathrm{X}$ & $\mathrm{X}$ & $\mathrm{X}$ & & $\mathrm{X}$ & $\mathrm{X}$ \\
\hline other & & & & & & & & & & & \\
\hline IR pumping & $\mathrm{X}$ & $\mathrm{X}$ & & & $\mathrm{X}$ & & & $\mathrm{X}$ & & & $\mathrm{X}$ \\
\hline
\end{tabular}

OBSERVATIONAL LINES

\begin{tabular}{|c|c|c|c|c|c|c|c|c|c|}
\hline self-consistent treatment with cooling & $\mathrm{X}$ & & $\mathrm{X}$ & & & & & & \\
\hline escape probability & $\mathrm{X}$ & & $\mathrm{X}$ & & $\mathrm{X}$ & $\mathrm{X}$ & $\mathrm{X}$ & $\mathrm{X}$ & $\mathrm{X}$ \\
\hline other & & $\mathrm{X}$ & & & $\mathrm{X}$ & & & & \\
\hline $\mathrm{H}_{2}$ & $\mathrm{X}$ & $\mathrm{X}$ & & & & & $\mathrm{X}$ & $\mathrm{x}$ & \\
\hline HD & & $\mathrm{X}$ & & & & & $\mathrm{x}$ & $\mathrm{X}$ & \\
\hline${ }^{12} \mathrm{CO}$ & $\mathrm{X}$ & $\mathrm{X}$ & $\mathrm{X}$ & $\mathrm{X}$ & $\mathrm{X}$ & $\mathrm{X}$ & & & $\mathrm{X}$ \\
\hline${ }^{13} \mathrm{CO}$ & $\mathrm{X}$ & $\mathrm{X}$ & & & $\mathrm{X}$ & & & & $\mathrm{X}$ \\
\hline $\mathrm{C}^{18} \mathrm{O}$ & & $\mathrm{X}$ & & & $\mathrm{x}$ & & & & \\
\hline${ }^{13} \mathrm{C}^{18} \mathrm{O}$ & & $\mathrm{X}$ & & & $\mathrm{X}$ & & & & \\
\hline [OI] & $\mathrm{X}$ & $\mathrm{X}$ & $\mathrm{X}$ & $\mathrm{X}$ & $\mathrm{x}$ & $\mathrm{X}$ & $\mathrm{X}$ & $\mathrm{X}$ & $\mathrm{X}$ \\
\hline [CII] & $\mathrm{X}$ & $\mathrm{X}$ & $\mathrm{X}$ & $\mathrm{X}$ & $\mathrm{X}$ & $\mathrm{X}$ & $\mathrm{X}$ & $\mathrm{X}$ & $\mathrm{X}$ \\
\hline [CI $]$ & $\mathrm{X}$ & $\mathrm{X}$ & $\mathrm{X}$ & $\mathrm{X}$ & $\mathrm{x}$ & $\mathrm{X}$ & $\mathrm{X}$ & $\mathrm{X}$ & $\mathrm{X}$ \\
\hline $\mathrm{Si}^{+}$ & $\mathrm{X}$ & $\mathrm{X}$ & & $\mathrm{X}$ & & & & & $\mathrm{X}$ \\
\hline$\overline{\mathrm{CS}}$ & & $\mathrm{X}$ & & & & & & & $\mathrm{X}$ \\
\hline $\mathrm{H}_{2} \mathrm{O}$ & & & & $\mathrm{X}$ & & & & & \\
\hline \multicolumn{10}{|l|}{$\mathrm{H}_{2}^{18} \mathrm{O}$} \\
\hline $\mathrm{HCO}^{+}$ & & $\mathrm{X}$ & & $\mathrm{X}$ & $\mathrm{X}$ & & & & $\mathrm{X}$ \\
\hline $\mathrm{OH}$ & & & & $\mathrm{X}$ & & & & & \\
\hline$[\mathrm{SiI}]$ & $\mathrm{x}$ & & & $\mathrm{X}$ & $\mathrm{X}$ & & & & \\
\hline [SI],[SII] & $\mathrm{X}$ & & & $\mathrm{X}$ & $\mathrm{x}$ & & & & $\mathrm{X}$ \\
\hline
\end{tabular}


Table A.1. continued.

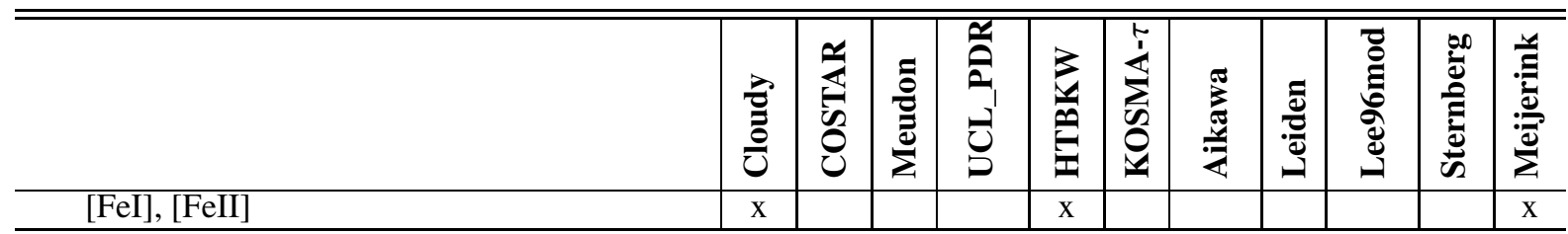

COMPUTED LINE PROPERTIES

\begin{tabular}{|c|c|c|c|c|c|c|c|}
\hline resolved line profile & & $\mathrm{X}$ & & $\mathrm{X}$ & $\mathrm{X}$ & $\mathrm{X}$ & \\
\hline $\begin{array}{l}\text { continuum rad./rad transfer in UV } \\
\text { line center intensities }\end{array}$ & $\begin{array}{l}\mathrm{X} \\
\mathrm{x}\end{array}$ & $\begin{array}{l}\mathrm{X} \\
\mathrm{X}\end{array}$ & & & $\mathrm{x}$ & $\mathrm{x}$ & \\
\hline line integrated intensities & $\mathrm{X}$ & $\mathrm{X}$ & $\mathrm{X}$ & $\mathrm{X}$ & $\mathrm{X}$ & $\mathrm{X}$ & $\mathrm{X}$ \\
\hline optical depths & $\mathrm{X}$ & $\mathrm{X}$ & $\mathrm{X}$ & $\mathrm{X}$ & $\mathrm{X}$ & $\mathrm{X}$ & $\mathrm{X}$ \\
\hline Gaussian line profile & $\mathrm{x}$ & $\mathrm{X}$ & & $\mathrm{X}$ & $\mathrm{x}$ & $\mathrm{X}$ & $\mathrm{X}$ \\
\hline box line profile & & & & & & & \\
\hline turbulence included & $\mathrm{X}$ & $\mathrm{X}$ & & & $\mathrm{X}$ & & $\mathrm{X}$ \\
\hline
\end{tabular}

COLLISIONS

\begin{tabular}{|c|c|c|c|c|c|c|c|c|c|}
\hline$\overline{\mathrm{H}-\mathrm{H}}$ & $\mathrm{x}$ & & & & $\mathrm{X}$ & & & $\mathrm{x}$ & \\
\hline $\mathrm{H}_{2}-\mathrm{H}$ & $\mathrm{X}$ & & $\mathrm{X}$ & $\mathrm{x}$ & $\mathrm{X}$ & & $\mathrm{X}$ & $\mathrm{X}$ & $\mathrm{x}$ \\
\hline $\mathrm{H}_{2}-\mathrm{H}+$ & $\mathrm{X}$ & & $\mathrm{X}$ & & & & & $\mathrm{X}$ & \\
\hline $\mathrm{H}_{2}-\mathrm{e}$ & $\mathrm{X}$ & & $\mathrm{X}$ & & $\mathrm{X}$ & & & $\mathrm{X}$ & \\
\hline $\mathrm{H}_{2}-\mathrm{H}_{2}$ & $\mathrm{X}$ & & $\mathrm{X}$ & & $\mathrm{X}$ & & $\mathrm{x}$ & $\mathrm{X}$ & $\mathrm{x}$ \\
\hline $\mathrm{CO}-\mathrm{H}$ & $\mathrm{X}$ & $\mathrm{X}$ & $\mathrm{X}$ & $\mathrm{X}$ & $\mathrm{X}$ & & $\mathrm{x}$ & & $\mathrm{x}$ \\
\hline $\mathrm{CO}-\mathrm{H}_{2}$ & $\mathrm{X}$ & $\mathrm{X}$ & $\mathrm{X}$ & $\mathrm{x}$ & $\mathrm{X}$ & $\mathrm{x}$ & $\mathrm{X}$ & & $\mathrm{X}$ \\
\hline CO-e & $\mathrm{X}$ & $\mathrm{X}$ & & $\mathrm{X}$ & & $\mathrm{X}$ & & & $\mathrm{X}$ \\
\hline $\mathrm{CO}-\mathrm{He}$ & & & $\mathrm{X}$ & $\mathrm{x}$ & & & & & $\mathrm{X}$ \\
\hline $\mathrm{C}-\mathrm{H}$ & $\mathrm{X}$ & $\mathrm{X}$ & $\mathrm{X}$ & $\mathrm{X}$ & $\mathrm{X}$ & $\mathrm{x}$ & $\mathrm{x}$ & $\mathrm{x}$ & $\mathrm{X}$ \\
\hline $\mathrm{C}-\mathrm{H}_{2}$ & $\mathrm{X}$ & $\mathrm{X}$ & $\mathrm{X}$ & $\mathrm{X}$ & $\mathrm{X}$ & $\mathrm{X}$ & $\mathrm{X}$ & & $\mathrm{X}$ \\
\hline C-e & $\mathrm{X}$ & & $\mathrm{X}$ & $\mathrm{X}$ & & & & & $\mathrm{X}$ \\
\hline $\mathrm{C}-\mathrm{He}$ & $\mathrm{X}$ & & $\mathrm{X}$ & $\mathrm{X}$ & & & & & \\
\hline \multicolumn{10}{|l|}{$\mathrm{C}-\mathrm{H}_{2} \mathrm{O}$} \\
\hline $\mathrm{C}^{+}-\mathrm{H}$ & $\mathrm{x}$ & $\mathrm{x}$ & $\mathrm{x}$ & $\mathrm{X}$ & $\mathrm{X}$ & & $\mathrm{X}$ & & $\mathrm{x}$ \\
\hline $\mathrm{C}^{+}-\mathrm{H}_{2}$ & $\mathrm{X}$ & $\mathrm{X}$ & $\mathrm{X}$ & $\mathrm{x}$ & $\mathrm{X}$ & $\mathrm{x}$ & $\mathrm{X}$ & & $\mathrm{X}$ \\
\hline $\mathrm{C}^{+}-\mathrm{e}$ & $\mathrm{X}$ & $\mathrm{X}$ & & $\mathrm{X}$ & $\mathrm{X}$ & $\mathrm{x}$ & $\mathrm{X}$ & & $\mathrm{X}$ \\
\hline $\mathrm{O}-\mathrm{H}$ & $\mathrm{X}$ & $\mathrm{X}$ & $\mathrm{x}$ & $\mathrm{X}$ & $\mathrm{X}$ & $\mathrm{X}$ & $\mathrm{X}$ & $x$ & $\mathrm{X}$ \\
\hline $\mathrm{O}-\mathrm{H}_{2}$ & $\mathrm{X}$ & $\mathrm{X}$ & $\mathrm{X}$ & $\mathrm{X}$ & $\mathrm{X}$ & $\mathrm{x}$ & $\mathrm{X}$ & $\mathrm{X}$ & $\mathrm{X}$ \\
\hline $\mathrm{O}-\mathrm{H}+$ & $\mathrm{X}$ & & $\mathrm{X}$ & $\mathrm{X}$ & & & $\mathrm{X}$ & & \\
\hline $\mathrm{O}-\mathrm{e}$ & $\mathrm{X}$ & $\mathrm{X}$ & $\mathrm{X}$ & $\mathrm{X}$ & $\mathrm{X}$ & & & & \\
\hline $\mathrm{O}-\mathrm{He}$ & $\mathrm{X}$ & & $\mathrm{X}$ & $\mathrm{X}$ & & & & & \\
\hline \multicolumn{10}{|l|}{$\overline{\mathrm{OH}}-\mathrm{H}$} \\
\hline \multicolumn{10}{|l|}{$\mathrm{OH}-\mathrm{He}$} \\
\hline $\mathrm{OH}-\mathrm{H}_{2}$ & & & & & $\mathrm{X}$ & $\mathrm{x}$ & & & \\
\hline $\mathrm{H}^{-}-\mathrm{H}$ & $\mathrm{X}$ & & & & & & & & \\
\hline \multicolumn{10}{|l|}{$\mathrm{H}_{2} \mathrm{O}-\mathrm{e}$} \\
\hline $\mathrm{H}_{2} \mathrm{O}-\mathrm{H}$ & & & & & & & & & $\mathrm{x}$ \\
\hline $\mathrm{H}_{2} \mathrm{O}-\mathrm{H}_{2}$ & & & & & $\mathrm{x}$ & & & & $\mathrm{X}$ \\
\hline \multicolumn{10}{|l|}{$\mathrm{H}_{2} \mathrm{O}-\mathrm{O}$} \\
\hline dust - $\mathrm{H} / \mathrm{H}_{2}$ & $\mathrm{x}$ & & & & $\mathrm{X}$ & & & & \\
\hline dust-any & $\mathrm{X}$ & & & & & & & & \\
\hline $\mathrm{Si}^{+}-\mathrm{H}$ & $\mathrm{X}$ & & $\mathrm{X}$ & & & $\mathrm{x}$ & & & \\
\hline $\mathrm{HD}-\mathrm{H}$ & & & $\mathrm{X}$ & & & & & & \\
\hline $\mathrm{HD}-\mathrm{H}_{2}$ & & & $\mathrm{X}$ & & & & & & \\
\hline PAH-any & $\mathrm{X}$ & & & & $\mathrm{X}$ & & & & \\
\hline
\end{tabular}

\section{OUTPUT}

\begin{tabular}{|c|c|c|c|c|c|c|c|c|c|c|c|}
\hline abundance profile over $\left(A_{V} /\right.$ depth $)$ & $\mathrm{x}$ & $\mathrm{X}$ & $\mathrm{x}$ & $\mathrm{x}$ & $\mathrm{x}$ & $\mathrm{x}$ & $\mathrm{x}$ & $\mathrm{x}$ & $\mathrm{x}$ & $\mathrm{x}$ & $\mathrm{X}$ \\
\hline column density over $\left(A_{V} /\right.$ depth $)$ & $\mathrm{X}$ & $\mathrm{X}$ & $\mathrm{X}$ & $\mathrm{X}$ & & $\mathrm{X}$ & & & & $\mathrm{x}$ & $\mathrm{X}$ \\
\hline temperature profile over $\left(A_{V} /\right.$ depth $)$ & $\mathrm{x}$ & $\mathrm{X}$ & $\mathrm{X}$ & $\mathrm{X}$ & $\mathrm{x}$ & $\mathrm{x}$ & $\mathrm{x}$ & $\mathrm{x}$ & & $\mathrm{x}$ & $\mathrm{X}$ \\
\hline emitted intensities & $\mathrm{x}$ & & $\mathrm{x}$ & $\mathrm{X}$ & $\mathrm{x}$ & $\mathrm{x}$ & & $\mathrm{x}$ & & $\mathrm{x}$ & $\mathrm{X}$ \\
\hline
\end{tabular}


Table A.1. continued.

\begin{tabular}{|c|c|c|c|c|c|c|c|c|c|c|c|}
\hline & $\frac{\vec{\theta}}{0}$ & 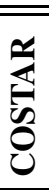 & 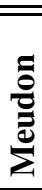 & 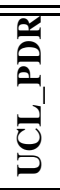 & $\frac{1}{3}$ & $\sum_{\substack{\infty \\
0}}^{5}$ & 菊 & 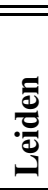 & 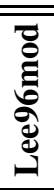 & 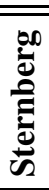 & 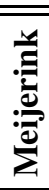 \\
\hline opacities at line center & & & $\mathrm{x}$ & & $\mathrm{x}$ & $\mathrm{X}$ & & $\mathrm{x}$ & & $\mathrm{X}$ & $\mathrm{X}$ \\
\hline heating and cooling rates over ( $A_{V} /$ depth $)$ & $\mathrm{x}$ & & $\mathrm{X}$ & $\mathrm{x}$ & & $\mathrm{X}$ & & $\mathrm{x}$ & & $\mathrm{x}$ & $\mathrm{x}$ \\
\hline chemical rates over $\left(A_{V} /\right.$ depth $)$ & & & $\mathrm{X}$ & $\mathrm{x}$ & & $\mathrm{x}$ & & $\mathrm{X}$ & & $\mathrm{X}$ & $\mathrm{x}$ \\
\hline excitation diagram of $\mathrm{H}_{2}$ & $\mathrm{x}$ & & $\mathrm{x}$ & & & & & $\mathrm{X}$ & & $\mathrm{x}$ & \\
\hline
\end{tabular}

